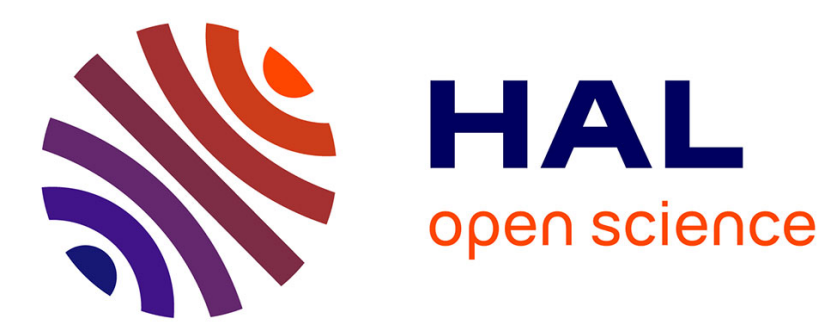

\title{
Tail dimension reduction for extreme quantile estimation Laurent Gardes
}

\section{To cite this version:}

Laurent Gardes. Tail dimension reduction for extreme quantile estimation. Extremes, 2018, 21 (1), pp.57-95. 10.1007/s10687-017-0300-x . hal-01322374v2

\section{HAL Id: hal-01322374 \\ https://hal.science/hal-01322374v2}

Submitted on 21 Jun 2017

HAL is a multi-disciplinary open access archive for the deposit and dissemination of scientific research documents, whether they are published or not. The documents may come from teaching and research institutions in France or abroad, or from public or private research centers.
L'archive ouverte pluridisciplinaire HAL, est destinée au dépôt et à la diffusion de documents scientifiques de niveau recherche, publiés ou non, émanant des établissements d'enseignement et de recherche français ou étrangers, des laboratoires publics ou privés. 


\title{
Tail dimension reduction for extreme quantile estimation
}

\author{
Laurent Gardes \\ Université de Strasbourg, CNRS, IRMA UMR 7501, \\ F-67000 Strasbourg, France. \\ gardes@unistra.fr
}

\begin{abstract}
In a regression context where a response variable $Y \in \mathbb{R}$ is recorded with a covariate $X \in \mathbb{R}^{p}$, two situations can occur simultaneously: (a) we are interested in the tail of the conditional distribution and not on the central part of the distribution and (b) the number $p$ of regressors is large. To our knowledge, these two situations have only been considered separately in the literature. The aim of this paper is to propose a new dimension reduction approach adapted to the tail of the distribution in order to propose an efficient conditional extreme quantile estimator when the dimension $p$ is large. The results are illustrated on simulated data and on a real dataset.
\end{abstract}

Keywords - Regression, extreme quantile, dimension reduction, kernel smoothing.

AMS Subject classifications - 62G32, 62G08, 62G05, 62G20.

\section{Introduction}

This work takes place in a regression context where a real response variable $Y$ is recorded with a random vector $X \in E \subset \mathbb{R}^{p}$ of explanatory variables. In the literature, several ways for examining how the distribution of $Y$ is influenced by the regressor $X$ have been considered. The most common approach summarize the relationship between $Y$ and $X$ by the regression function $\mathbb{E}(Y \mid X)$ which is the conditional expectation of $Y$ given $X$. Several estimators of the regression function are available, the probably most known being the kernel estimator introduced independently by Nadaraya [29] and Watson [35]. In the same spirit, one can also mention the estimator introduced by Gasser and Müller [20]. Another way to understand the link between $Y$ and $X$ is to use a conditional quantile of fixed order $1-\alpha \in(0,1)$. For instance, Koenker and Basset [26] introduced the notion of quantile regression assuming that the conditional quantile of $Y$ given $X$ is a linear combination of the explanatory variables. This approach has the advantage of being more robust against outliers than the regression function. Concerning the estimation of conditional quantile, local linear approaches were considered by $\mathrm{Yu}$ and Jones [39] while a fully nonparametric estimator can be found in the paper of Chaudhuri [3].

In many applications such as climatology, finance, insurance to name a few, two situations can occur simultaneously in a regression context.

(a) We are interested in the tail distribution of $Y$ given $X$ instead of the central part of the conditional distribution. In this case, regression function and conditional quantile of fixed order $1-\alpha$ are clearly irrelevant tools. 
(b) The dimension $p$ of the regressor is large. In this situation, inference on the conditional distribution of $Y$ given $X$ becomes difficult since the space is sparsely populated by data points. This is the well known curse of dimensionality problem.

A motivating example is the study of the influence of various pollutants (sulphur dioxide, nitrogen dioxide, carbon monoxide, ...) and weather conditions (temperature, humidity, ...) on extreme values of ozone concentration (see Section 5). Another example can be found in hydrology where the understanding of the influence of the geographical position and the altitude on return periods of large amount of rain is a problem of primary interest (see Gardes and Girard [17]). Despite this large range of applications, situations (a) and (b) mentioned before have been considered separately in the literature.

To make inference on the tail distribution of $Y$ given $X$, one solution is to use a conditional quantile of order $1-\alpha_{n}$ where $\alpha_{n} \rightarrow 0$ as the sample size $n$ goes to infinity. Such a quantile is said to be extreme. Estimation of conditional extreme quantiles has been considered by many authors. One common approach consists to fit a parametric model for exceedances over a high threshold (see Davison and Smith [9] and Northrop and Jonathan [30]). In Davison and Ramesh [10], a local likelihood smoothing procedure is considered for the estimation of a conditional generalized extreme value distribution and Eastoe and Tawn [12] propose to model the covariate effect by a Box-Cox location-scale model. A nonparametric estimation procedure is proposed by Daouia et al. ([7] and [8]) and Gardes and Girard [18].

To deal with high dimensional covariates, a classical method is to assume the existence of a $p \times q$ full rank matrix $B$ (with $q<p$ ) such that the conditional distributions of $Y$ given $B^{\top} X$ and $Y$ given $X$ are the same. In others words, it is assumed that $X$ and $Y$ are independent conditionally on $B^{\top} X$ (in symbols $X \Perp Y \mid B^{\top} X$ ). For a comprehensive discussion on conditional independence see Basu and Pereira [2]. In the literature, this model is referred to the multipleindex model (single-index model if $q=1$ ) and the subspace spanned by the columns of $B$ is called the Dimension Reduction (DR) subspace. Among the contributions on the estimation of the DR subspace, one can cite the Sliced Inverse Regression (SIR) method introduced by Li [27], the Slice Average Variance Estimation (SAVE) method proposed by Cook and Weisberg [6] and the Principal Hessian Directions (PHD) method (see Li [28]). The existence of a DR subspace is assumed by many authors in order to study the link between $Y$ and the explanatory variables $X$. For instance, to estimate conditional quantiles of fixed order, Wu et al. [37] use a single-index model while a combination of SIR and kernel estimation is considered by Gannoun et al. [15].

To our knowledge, the use of adapted dimension reduction methods for estimating conditional extreme quantiles has not been considered yet in the literature. One can mention the recent paper of Russel et al. [32] where the relationship between $Y$ and $X$ is summarized by the link between $Y$ and a linear combination of the explanatory covariates. This combination is obtained by optimizing the tail dependence with the response variable. Note that this method is based on the assumption that the random vector $(X, Y)$ is regularly varying while no particular condition on $X$ is required in our approach.

In the present paper we first adapt the classical definition of conditional independence to an extreme value context. More specifically, we introduce the notion of Tail Conditional Independence (TCI) of $Y$ and $X$ given $Z$. Roughly speaking, TCI means that the tail distribution of $Y$ given $(X, Z)$ is asymptotically equivalent to the one of $Y$ given $Z$. This new definition permits us to deal with situations where the covariate dimension can be reduced only in the tail of the distribution. 
Next, the notion of Tail Dimension Reduction (TDR) subspace is introduced. The TDR subspace is spanned by a $p \times q$ full rank matrix $B$ such that $Y$ and $X$ are tail conditionally independent given $B^{\top} X$. Note that for any regular $q \times q$ matrix $D$, the subspace spanned by $B$ is also the subspace spanned by $B D$. To avoid this misspecification issue, the matrix $B$ spanning the TDR subspace is taken in the set $\mathcal{B}$ where $B \in \mathcal{B}$ if the $q$ columns of $B$ are the first normalized $q$ linearly independent columns of the orthogonal projection matrix on the subspace spanned by $B$. Taking advantage of the existence of a TDR subspace, a kernel-based statistic is then proposed as a first estimator of conditional extreme quantiles. Unfortunately, this estimator is only of theoretical interest since it depends on the unknown direction $B$ of the TDR subspace. Estimation of $B$ is thus considered leading to the definition of a more useful conditional extreme quantile estimator.

The paper is organized as follows. Tail Conditional Independence and Tail Dimension Reduction subspace are defined in Section 2. In Section 3, assuming the existence of a Tail Dimension Reduction subspace, the estimation of conditional extreme quantiles is addressed. Finite sample properties are investigated through a simulation study in Section 4. Note that, due the computational cost in the estimation of $B$ when $q>1$ (see end of Section 3.2), only the case $q=1$ is considered. Our estimation procedure is applied to study the influence of various pollutants on ozone concentration in Section 5. Proofs are postponed to the appendix.

\section{Tail Conditional Independence and Tail Dimension Reduc- tion subspace}

\subsection{Definition of Tail Conditional Independence}

Let $(X, Y, Z) \in \mathbb{R}^{p} \times \mathbb{R} \times \mathbb{R}^{q}$ be a random vector defined on a probability space $(\Omega, \mathcal{F}, \mathbb{P})$. The goal of this section is to introduce the notion of Tail Conditional Independence (TCI) of $Y$ and $X$ given $Z$. First, let us give some notations used in all what follows. For any random variable $W:(\Omega, \mathcal{F}, \mathbb{P}) \mapsto\left(\mathbb{R}^{m}, \mathcal{B}\left(\mathbb{R}^{m}\right)\right)$ where $m \in \mathbb{N}^{*}$, let $\operatorname{supp}(W)$ be the support of its distribution. We denote by $\mathbb{P}(\cdot \mid W=\cdot): \mathcal{F} \times \operatorname{supp}(W) \mapsto[0,1]$ a regular version of the conditional expectation $\mathbb{E}\left(\mathbb{I}_{\{\cdot\}} \mid W\right)$ where the dot denotes any element of $\mathcal{F}$ and $\mathbb{I}_{\{\cdot\}}$ is the indicator function. Finally, the conditional quantile of $Y$ given $W$ of order $\alpha \in[0,1]$ is the measurable function $Q(\alpha \mid W=\cdot):=$

$\inf \{y \in \overline{\mathbb{R}} ; \mathbb{P}(Y>y \mid W=\cdot) \leq \alpha\}$ where $\overline{\mathbb{R}}$ denotes the extended real number line. The TCI property is defined below.

Definition 1. The random variable $Y$ is tail conditionally independent of $X$ given $Z$ (in symbols $Y \underset{\sim}{\sim} X \mid Z)$ if for all $\varepsilon>0$ there exists $\kappa>0$ such that for all $\delta \in(0, \kappa]$,

$$
\mathbb{P}\left[\left|\frac{\mathbb{P}\left(Y>\mathcal{Y}_{\delta}(Z) \mid X, Z\right)}{\mathbb{P}\left(Y>\mathcal{Y}_{\delta}(Z) \mid Z\right)}-1\right| \leq \varepsilon\right]=1
$$

where for $\delta>0, \mathcal{Y}_{\delta}(Z=\cdot)$ is a measurable function defined on $\operatorname{supp}(Z)$ and given by $\mathcal{Y}_{\delta}(Z=\cdot):=$ $Q(0 \mid Z=\cdot)-\delta$ if $Q(0 \mid Z=\cdot)<+\infty$ and $\mathcal{Y}_{\delta}(Z=\cdot):=\delta^{-1}$ if $Q(0 \mid Z=\cdot)=+\infty$.

Note that, as a direct consequence of (1), if $Y \underset{\sim}{\Perp} X \mid Z$ then $Q(0 \mid X, Z)=Q(0 \mid Z)$ a.s. that is to say that the distributions of $Y$ given $(X, Z)$ and $Y$ given $Z$ share the same right endpoint.

For a better understanding of Definition 1, one can remark that if $Y \underset{\sim}{\Perp} X \mid Z$, there exists a Borel 
set $\mathcal{A} \in \mathcal{B}\left(\mathbb{R}^{p}\right) \otimes \mathcal{B}\left(\mathbb{R}^{q}\right)$ with $\mathbb{P}[(X, Z) \in \mathcal{A}]=1$ such that for all $(x, z) \in \mathcal{A}$,

$$
\lim _{y \uparrow Q(0, Z=z)} \frac{\mathbb{P}(Y>y \mid X=x, Z=z)}{\mathbb{P}(Y>y \mid Z=z)}=1 .
$$

Hence, if $Y \underset{\sim}{\Perp} X \mid Z$, the conditional tail distribution of $Y$ given $(X, Z)$ is asymptotically equivalent to the one of $Y$ given $Z$ and thus, inference on the tail of $Y$ given $(X, Z)$ can be achieved without the information carried by $X$. This property can be of high interest in practice if the dimension of $Z$ is much smaller than that of $X$ (see the next section).

The TCI property is obviously less restrictive than the classical conditional independence property since the last one assumes that $\mathbb{P}(Y>y \mid X, Z)=\mathbb{P}(Y>y \mid Z)$ a.s. for all $y \in \mathbb{R}$. Note also that the conditional independence property is symmetric (i.e. $X \Perp Y|Z \Leftrightarrow Y \Perp X| Z$ ) but not the tail conditional independence property.

As it is the case for the conditional independence property, the TCI property can be characterized in different equivalent ways. This is the purpose of the next result.

Theorem 1. The following statements are equivalent:

(i) $Y \underset{\sim}{\Perp} X \mid Z$.

(ii) For all $\varepsilon>0$ there exists $\kappa>0$ such that for all $\delta \in(0, \kappa]$ and for all non-zero bounded and positive measurable function $h(\cdot)$,

$$
\mathbb{P}\left[\left|\frac{\mathbb{E}\left(\mathbb{I}_{\left\{Y>\mathcal{Y}_{\delta}(Z)\right\}} h(X) \mid Z\right)}{\mathbb{P}\left(Y>\mathcal{Y}_{\delta}(Z) \mid Z\right) \mathbb{E}(h(X) \mid Z)}-1\right| \leq \varepsilon\right]=1 .
$$

(iii) For all $\delta>0, \mathbb{P}\left(Y>\mathcal{Y}_{\delta}(Z) \mid X, Z\right)=s_{\delta}(Z)\left(1+\eta_{\delta}(X, Z)\right)$ a.s., where $s_{\delta}: \operatorname{supp}(Z) \mapsto \mathbb{R}$ and $\eta_{\delta}: \operatorname{supp}(X, Z) \mapsto \mathbb{R}$ are two measurable functions and, for all $\varepsilon \in(0,1)$, there exists $\kappa>0$ such that for all $\delta \in(0, \kappa], \mathbb{P}\left[\left|\eta_{\delta}(X, Z)\right| \leq \varepsilon\right]=1$.

Note that uniform convergence in (1) (i.e. the fact that $\kappa$ does not depend on the values of the random vector $(X, Z))$ is essential to prove the second and third statements in Theorem 1 . Furthermore, since the conditional expectation is almost surely unique, Theorem 1 can not be established if we ask that (2) holds everywhere.

The second statement gives us another way to understand the TCI property. It entails that for all bounded and positive measurable function $h(\cdot)$, there exists a Borel set $\mathcal{A} \in \mathcal{B}\left(\mathbb{R}^{q}\right)$ with $\mathbb{P}(Z \in \mathcal{A})=1$ such that for all $z \in \mathcal{A}, \mathbb{E}\left(\mathbb{I}_{\{Y>y\}} h(X) \mid Z=z\right) \sim \mathbb{P}(Y>y \mid Z=z) \mathbb{E}(h(X) \mid Z=z)$ as $y \uparrow Q(0 \mid Z=z)$. In the particular situation considered later where $Z=B^{\top} X$ with $B$ a full rank $p \times q$ matrix $(q<p)$, this property will be the starting point for the estimation of $B$.

The third statement is a very useful tool for anyone who wants to propose conditional distributions of $Y$ given $(X, Z)$ satisfying Definition 1. Some examples are given hereafter.

Example 1. Let $\mu_{1}(\cdot \mid(X, Z)=\cdot): \mathcal{B}(\mathbb{R}) \times \operatorname{supp}(X, Z) \mapsto([0,1], \mathcal{B}([0,1]))$ and $\mu_{2}(\cdot \mid Z=\cdot): \mathcal{B}(\mathbb{R}) \times$ $\operatorname{supp}(Z) \mapsto([0,1], \mathcal{B}([0,1]))$ be two functions such that for all $(x, z) \in \operatorname{supp}(X, Z), \mu_{1}(\cdot \mid(X, Z)=$ $(x, z))$ and $\mu_{2}(\cdot \mid Z=z)$ are two probability measures on $(\mathbb{R}, \mathcal{B}(\mathbb{R}))$ and, for all $A \in \mathcal{B}(\mathbb{R})$, the functions $\mu_{1}(A \mid(X, Z)=\cdot)$ and $\mu_{2}(A \mid Z=\cdot)$ are measurable. We assume in addition that uniformly on $(x, z) \in \operatorname{supp}(X, Z)$,

$$
\lim _{\delta \rightarrow 0} \frac{\mu_{1}\left(I_{\delta}(z) \mid(X, Z)=(x, z)\right)}{\mu_{2}\left(I_{\delta}(z) \mid Z=z\right)}=0 .
$$


where $I_{\delta}(z)=\left[\mathcal{Y}_{\delta}(Z=z), \infty\right)$. Regardless of the distribution of $(X, Z)$, if the conditional distribution of $Y$ given $(X, Z)$ is the mixture distribution defined by

$$
\mathbb{P}(Y \in \cdot \mid X, Z)=\theta(Z) \mu_{1}(\cdot \mid X, Z)+(1-\theta(Z)) \mu_{2}(\cdot \mid Z) \text { a.s. }
$$

where $\theta(\cdot)$ is a $[0,1)$-valued measurable function, then, from Theorem 1 point (iii), it is easy to see that $Y \stackrel{\Perp}{\sim} X \mid Z$.

Example 2. Consider the semi-parametric model

$$
\mathbb{P}(Y>y \mid X)=y^{-\exp \left(b_{0}^{\top} X\right)} \mathcal{L}(y \mid X) \text { a.s. }
$$

where $b_{0} \in \mathbb{R}^{p}$ and $\mathcal{L}(\cdot \mid X=\cdot): \mathbb{R} \times \operatorname{supp}(X) \mapsto(0, \infty)$ is a function such that for all $t>0$ and $x \in \operatorname{supp}(X), \mathcal{L}(t y \mid X=x) / \mathcal{L}(y \mid X=x) \rightarrow 1$ as $y \rightarrow \infty$. Note that this model was introduced by Wang and Tsai [34] where a maximum likelihood method to estimate $b_{0}$ is considered. If we assume that $\mathcal{L}(y \mid X=x)$ converges to $c\left(b_{0}^{\top} x\right)$ uniformly on $x \in \operatorname{supp}(X)$ as $y \rightarrow \infty$ where $c(\cdot)$ is a positive and measurable function then, using the third statement of Theorem 1, it is easy to check that $Y \underset{\sim}{\Perp} X \mid b_{0}^{\top} X$.

\subsection{Tail Dimension Reduction subspace}

Using the notion of Tail Conditional Independence presented in the previous section, we give now the definition of a TDR subspace. In what follows, for a full rank $p \times q$ matrix $B$ with $q<p$, the space spanned by the columns of $B$ is denoted $\mathcal{S}(B)$.

Definition 2. If there exists a full rank $p \times q$ matrix $B_{0} \in \mathcal{B}$ with $q<p$ such that $Y \underset{\sim}{\sim} X \mid B_{0}^{\top} X$ then $\mathcal{S}\left(B_{0}\right)$ is a Tail Dimension Reduction (TDR) subspace for $Y$ given $X$.

The TDR subspace is an adaptation of the DR subspace introduced by Li [27]. Recall that since $B_{0} \in \mathcal{B}$, the matrix $B_{0}$ spanning the TDR subspace is unique. In particular its columns are orthogonal unit vectors. Roughly speaking, if there exists a TDR subspace $\mathcal{S}\left(B_{0}\right)$, the tail of the conditional distribution of $Y$ given $X$ can be reasonably approximated by the tail of the conditional distribution of $Y$ given $B_{0}^{\top} X$. Obviously, for any random vector $(X, Y), \mathcal{S}\left(I_{p}\right)=\mathbb{R}^{p}$ is a TDR subspace and thus a TDR subspace is not unique. Since our goal is to reduce the dimension, the notion of minimum TDR subspace is defined below by analogy with the definition of the minimum DR subspace (see for instance [4]).

Definition 3. For a full rank matrix $B_{0} \in \mathcal{B}$, the subspace $\mathcal{S}\left(B_{0}\right)$ is a minimum TDR subspace if its dimension is less than or equal to the dimension of any other TDR subspace.

\section{Extreme quantile estimation under a TDR model}

Let $(X, Y) \in \mathbb{R}^{p} \times \mathbb{R}$ be a random vector defined on the probability space $(\Omega, \mathcal{F}, \mathbb{P})$. In what follows, the distribution of $(X, Y)$ is assumed to be absolutely continuous with respect to the Lebesgue measure. A probability distribution function of $X$ is denoted by $f_{X}(\cdot)$ and its support is given by $\operatorname{supp}(X):=\left\{x \in \mathbb{R}^{p} ; f_{X}(x)>0\right\}$ which is assumed to be an open set.

The aim of this section is to propose an estimator of the conditional quantile $Q(\alpha \mid X)$ when $\alpha$ is close to 0 (conditional extreme quantile) and when the dimension $p$ of the covariate $X$ is large. Without assuming the existence of a minimum TDR subspace, this question has already been 
investigated by several authors (see for instance Araújo Santos et al. [1], Daouia et al. [7], Gardes and Girard [18] among many others). Unfortunately, these estimators often fail to approximate correctly the conditional extreme quantile in a large dimension setting since in this situation, the space $\operatorname{supp}(X)$ is only sparsely populated by data points. As a consequence, only few points can be reasonably considered to estimate $Q\left(\alpha_{n} \mid X=x\right)$ and, unless the sample size is very large, classical estimators become inefficient for large values of $p$.

In this section, the existence of a minimum TDR subspace $\mathcal{S}\left(B_{0}\right)$ is assumed and a new kernel estimator of $Q\left(\alpha_{n} \mid X=x\right)$ is proposed. This new estimator is expected to be more efficient than classical kernel estimators for large values of $p$. In a preliminary step (see Section 3.1), we introduce a statistic $\widehat{Q}_{n}\left(\alpha_{n} \mid B_{0}, x\right)$ depending on the unknown direction $B_{0}$ and which is consistent for the estimation of $Q\left(\alpha_{n} \mid X=x\right)$. Obviously, this statistic is useless since in practice $B_{0}$ is unknown. An estimator of $B_{0}$ is thus proposed in Section 3.2 and the estimation of $Q\left(\alpha_{n} \mid X=x\right)$ is achieved by replacing the true direction $B_{0}$ by its estimated version in $\widehat{Q}_{n}\left(\alpha_{n} \mid B_{0}, x\right)$.

\subsection{Conditional extreme quantile estimation: the case $B_{0}$ known}

Given $n$ independent copies $\left(X_{1}, Y_{1}\right), \ldots,\left(X_{n}, Y_{n}\right)$ of the random vector $(X, Y)$, we are interested in the estimation of the conditional extreme quantile $Q\left(\alpha_{n} \mid X=x\right)$ for $x \in \operatorname{supp}(X)$ where $\alpha_{n} \in(0,1)$ converges to 0 as $n$ goes to infinity.

The first step is the estimation of the conditional survival function $\mathbb{P}(Y>y \mid X=x)$ for large values of $y$. Assuming the existence of a minimum TDR subspace $\mathcal{S}\left(B_{0}\right)$, the conditional survival function $\mathbb{P}(Y>y \mid X=x)$ can be approximated for $y$ large enough by $\mathbb{P}\left(Y>y \mid B_{0}^{\top} X=B_{0}^{\top} x\right)$. We thus propose the following statistic as an estimator of $\mathbb{P}(Y>y \mid X=x)$ :

$$
\widehat{S}_{n}\left(y \mid B_{0}, x\right):=\sum_{i=1}^{n} \mathbb{I}_{\left\{Y_{i}>y\right\}} K\left(H_{n}^{-1} B_{0}^{\top}\left(x-X_{i}\right)\right) / \sum_{i=1}^{n} K\left(H_{n}^{-1} B_{0}^{\top}\left(x-X_{i}\right)\right) .
$$

Here, $H_{n}$ is a sequence of $q \times q$ positive definite matrices and $K(\cdot)$ is a probability distribution function on $\mathbb{R}^{q}$. From now on, we assume that $K(\cdot)$ is bounded with support the unit ball of $\mathbb{R}^{q}$. Note that $\widehat{S}_{n}\left(y \mid B_{0}, x\right)$ is the classical kernel estimator of $\mathbb{P}\left(Y>y \mid B_{0}^{\top} X=B_{0}^{\top} x\right)$ that is considered here as an estimator of $\mathbb{P}(Y>y \mid X=x)$.

A first attempt to estimate $Q\left(\alpha_{n} \mid X=x\right)$ is to use the generalized inverse of $\widehat{S}_{n}\left(\cdot \mid B_{0}, x\right)$ leading to the statistic

$$
\widehat{Q}_{n}\left(\alpha_{n} \mid B_{0}, x\right):=\inf \left\{y ; \widehat{S}_{n}\left(y \mid B_{0}, x\right) \leq \alpha_{n}\right\} .
$$

Unfortunately, such an estimator fails to estimate extreme quantiles of order $\alpha_{n}$ as small as we like. Indeed, it is shown in Proposition 4 that the condition $n\left|H_{n}\right| \alpha_{n} \rightarrow \infty$, where $\left|H_{n}\right|$ stands for the determinant of $H_{n}$, is required to establish the consistency of (4). As a consequence, $Q\left(\alpha_{n} \mid X=x\right)$ cannot be consistently estimated by (4) when $\alpha_{n}$ is too small. To overcome this drawback, additional information on the tail distribution of $Y$ given $X$ is necessary.

Extended regular variation. In the unconditional case, when dealing with the right-tail of a real random variable $Y$, it is commonly assumed that $Y$ belongs to the maximum domain of attraction of an extreme value distribution (see Fisher and Tippett [13] and Gnedenko [21] for a definition). According to de Haan and Ferreira [22, Theorem 1.1.6], this is equivalent to assuming the existence of a positive auxiliary function $a_{Y}(\cdot)$ and a parameter $\gamma_{Y} \in \mathbb{R}$ such that for all $u>0$, $\left[Q_{Y}(u \alpha)-Q_{Y}(\alpha)\right] / a_{Y}\left(\alpha^{-1}\right) \rightarrow L_{\gamma_{Y}}(1 / u)$ as $\alpha \rightarrow 0$ where $Q_{Y}(\alpha)=\inf \{y ; \mathbb{P}(Y>y) \leq \alpha\}$ and with 
for all $v \geq 1$ and $s \in \mathbb{R}, L_{s}(v):=\int_{1}^{v} u^{s-1} d u$. The function $Q_{Y}(\cdot)$ is then said to be of extended regular variation.

The same kind of assumption is made in our conditional setting. We assume that for all $x \in$ $\operatorname{supp}(X)$, the function $Q(\cdot \mid X=x)$ is of extended regular variation i.e. that there exist a positive function $a(\cdot \mid x):(0, \infty) \mapsto(0, \infty)$ and a real-valued function $\gamma(\cdot)$ such that for all $x \in \operatorname{supp}(X)$ and $u>0$,

$$
\operatorname{ERV}(\alpha, u \mid x):=\left|\frac{Q(u \alpha \mid X=x)-Q(\alpha \mid X=x)}{a\left(\alpha^{-1} \mid x\right)}-L_{\gamma(x)}(1 / u)\right| \rightarrow 0,
$$

as $\alpha \rightarrow 0$. Note that this convergence holds locally uniformly on $u \in(0, \infty)$. The function $\gamma(\cdot)$ is referred to as the conditional extreme value index function. The function $a(\cdot \mid x)$, called the auxiliary function, is such that $a\left(\alpha^{-1} \mid x\right) / Q(\alpha \mid X=x)-\gamma_{+}(x) \rightarrow 0$ as $\alpha$ goes to 0 where $(\cdot)_{+}$and $(\cdot)_{-}$are the positive and negative part functions (see Fraga Alves et al. [14, Lemma 3.1]). Condition (5) is equivalent to assuming that the distribution function $\mathbb{P}(Y \leq \cdot \mid X=x)$ belongs to the maximum domain of attraction of an extreme value distribution with extreme value index $\gamma(x)$. Condition (5) is also satisfied for the conditional quantile of $Y$ given $B_{0}^{\top} X$ as it is shown in the following result.

Proposition 1. Let $B_{0} \in \mathcal{B}$ be a full rank matrix such that $\mathcal{S}\left(B_{0}\right)$ is a TDR subspace. If for all $x \in \operatorname{supp}(X)$, the conditional quantile $Q(\cdot \mid X=x)$ satisfies (5), there exist a Borel set $\mathcal{A} \in \mathcal{B}\left(\mathbb{R}^{p}\right)$ with $\mathbb{P}(X \in \mathcal{A})=1$, a positive function $\tilde{a}\left(\cdot \mid B_{0}^{\top} x\right)$ and a real-valued function $\tilde{\gamma}(\cdot)$ such that for all $u>0$ and $x \in \mathcal{A}$,

$$
\lim _{\alpha \rightarrow 0} \frac{Q\left(u \alpha \mid B_{0}^{\top} X=B_{0}^{\top} x\right)-Q\left(\alpha \mid B_{0}^{\top} X=B_{0}^{\top} x\right)}{\tilde{a}\left(\alpha^{-1} \mid B_{0}^{\top} x\right)}=L_{\tilde{\gamma}\left(B_{0}^{\top} x\right)}(1 / u) .
$$

In addition, for all $x \in \mathcal{A}, \gamma(x)=\tilde{\gamma}\left(B_{0}^{\top} x\right)$ and $\tilde{a}\left(\alpha^{-1} \mid B_{0}^{\top} x\right)=a\left(\alpha^{-1} \mid x\right)$.

To estimate $Q\left(\beta_{n} \mid X=x\right)$ for an arbitrary sequence $\left(\beta_{n}\right)$ converging to 0 , we start with (5) which suggests the approximation $Q\left(\beta_{n} \mid X=x\right) \approx Q\left(\alpha_{n} \mid X=x\right)+a\left(\alpha_{n}^{-1} \mid x\right) L_{\gamma(x)}\left(\alpha_{n} / \beta_{n}\right)$. The sequence $\left(\alpha_{n}\right)$ is chosen not too small so that $Q\left(\alpha_{n} \mid X=x\right)$ can be consistently estimated by the kernel estimator $\widehat{Q}_{n}\left(\alpha_{n} \mid B_{0}, x\right)$ defined in (4). Assuming as before that $\mathcal{S}\left(B_{0}\right)$ is a TDR subspace for a given full rank matrix $B_{0}$, an estimator of $Q\left(\beta_{n} \mid X=x\right)$ is thus given by

$$
\check{Q}_{n}\left(\beta_{n} \mid B_{0}, x\right):=\widehat{Q}_{n}\left(\alpha_{n} \mid B_{0}, x\right)+\widehat{a}_{n}\left(B_{0}, x\right) L_{\widehat{\gamma}_{n}\left(B_{0}, x\right)}\left(\alpha_{n} / \beta_{n}\right),
$$

where $\widehat{\gamma}_{n}\left(B_{0}, x\right)$ and $\widehat{a}_{n}\left(B_{0}, x\right)$ are consistent estimators of $\gamma(x)$ and $a\left(\alpha_{n}^{-1} \mid x\right)$. Before giving the expression of these two estimators, let us introduce some notations. For $\nu \in(0,1)$ and $\varphi(\cdot)$ a positive and bounded function on $[\nu, 1]$, let $\Psi(\cdot)$ be the decreasing function defined for $s \geq 0$ by $\Psi(s)=0$ and for $s \leq 0$ by

$$
\Psi(s):=\left(\int_{\nu}^{1} \varphi(u) L_{s}(1 / u) d u\right)^{2} / \int_{\nu}^{1} \varphi(u) L_{s}^{2}(1 / u) d u .
$$

The function $\Psi^{\leftarrow}(\cdot)$ given by $\Psi^{\leftarrow}(t)=\inf \{s ; \Psi(s) \leq t\}$ is the generalized inverse of $\Psi(\cdot)$. In addition, for all $\delta \in \mathbb{N}$, for all non-increasing right-continuous function $U(\cdot)$ and all $\alpha \in(0,1)$, let

$$
\mathcal{T}_{\alpha}^{(\delta)}(U):=\int_{\nu}^{1} \varphi(u)\left(\ln \frac{U(u \alpha)}{U(\alpha)}\right)^{\delta} d u /\left(\int_{\nu}^{1} \varphi(u) L_{0}(1 / u) d u\right)^{\delta} .
$$

The estimator $\widehat{\gamma}_{n}\left(B_{0}, x\right)$ is given by

$$
\begin{aligned}
\widehat{\gamma}_{n}\left(B_{0}, x\right) & :=\widehat{\gamma}_{n,+}\left(B_{0}, x\right)+\widehat{\gamma}_{n,-}\left(B_{0}, x\right) \\
& =\mathcal{T}_{\alpha_{n}}^{(1)}\left(\widehat{Q}_{n}\left(\cdot \mid B_{0}, x\right)\right)+\Psi^{\leftarrow}\left(\frac{\left[\mathcal{T}_{\alpha_{n}}^{(1)}\left(\widehat{Q}_{n}\left(\cdot \mid B_{0}, x\right)\right]^{2}\right.}{\mathcal{T}_{\alpha_{n}}^{(2)}\left(\widehat{Q}_{n}\left(\cdot \mid B_{0}, x\right)\right.}\right) .
\end{aligned}
$$


Note that this estimator belongs to the class of estimators introduced in Gardes [16]. Concerning the estimation of $a\left(\alpha_{n}^{-1} \mid x\right)$, we consider the statistic

$$
\widehat{a}_{n}\left(B_{0}, x\right)=\widetilde{\mathcal{T}}_{\alpha_{n}}\left(\widehat{Q}_{n}\left(\cdot \mid B_{0}, x\right) ; \widehat{\gamma}_{n,-}\left(B_{0}, x\right)\right),
$$

where $\widetilde{\mathcal{T}}_{\alpha}\left(U, \gamma_{-}\right)$is given for all non-increasing and right-continuous function $U(\cdot)$, for all $\gamma_{-} \leq 0$ and for all $\alpha \in(0,1)$ by

$$
\widetilde{\mathcal{T}}_{\alpha}\left(U, \gamma_{-}\right):=U(\alpha) \int_{\nu}^{1} \varphi(u) \ln \frac{U(u \alpha)}{U(\alpha)} d u / \int_{\nu}^{1} \varphi(u) L_{\gamma_{-}}(1 / u) d u .
$$

Note that the previous defined estimators depend on the choice of a parameter $\nu \in(0,1)$ and a positive and bounded function $\varphi(\cdot)$ on $[\nu, 1]$. In order to not overload the notations, this dependence has been omitted. Expressions of $\widehat{\gamma}_{n}\left(B_{0}, x\right)$ and $\widehat{a}_{n}\left(B_{0}, x\right)$ are motivated by the following result.

Proposition 2. Let $x \in \operatorname{supp}(X)$. If condition (5) holds then

$$
\begin{array}{ll} 
& \lim _{\alpha \rightarrow 0} \mathcal{T}_{\alpha}^{(1)}(Q(\cdot \mid X=x))=\gamma_{+}(x), \quad \lim _{\alpha \rightarrow 0} \frac{\left[\mathcal{T}_{\alpha}^{(1)}(Q(\cdot \mid X=x))\right]^{2}}{\mathcal{T}_{\alpha}^{(2)}(Q(\cdot \mid X=x))}=\Psi\left(\gamma_{-}(x)\right), \\
\text { and } \quad \lim _{\alpha \rightarrow 0} \frac{\widetilde{\mathcal{T}}_{\alpha}\left(Q(\cdot \mid X=x), \gamma_{-}(x)\right)}{a\left(\alpha^{-1} \mid x\right)}=1 .
\end{array}
$$

The proof of this Proposition is a direct consequence of [16, Lemma 3]. Its proof is thus omitted. The study of the asymptotic behavior of $\check{Q}_{n}\left(\beta_{n} \mid B_{0}, x\right)$ is done under the assumptions given below.

Condition on the TDR subspace. Recall that, from Theorem $1, \mathcal{S}\left(B_{0}\right)$ is a TDR subspace if and only if for all $\delta>0$ there exist two measurable functions $s_{\delta}(\cdot)$ and $\eta_{\delta}(\cdot)$ such that $\mathbb{P}\left(Y>\mathcal{Y}_{\delta}\left(B_{0}^{\top} X\right) \mid X\right)=s_{\delta}\left(B_{0}^{\top} X\right)\left(1+\eta_{\delta}(X)\right)$ almost surely. As shown in the proof of Theorem 1, (see equation (21)), the function $\eta_{\delta}(\cdot)$ controls the rate of convergence of the ratio $\mathbb{P}\left(Y>\mathcal{Y}_{\delta}\left(B_{0}^{\top} X\right) \mid X\right) / \mathbb{P}\left(Y>\mathcal{Y}_{\delta}\left(B_{0}^{\top} X\right) \mid B_{0}^{\top} X\right)$ to 1 . In the sequel, we assume that there exist $\kappa>0$ and a decreasing function $\bar{\eta}(\cdot)$ converging to 0 at infinity such that for all $\delta \in(0, \kappa]$

$$
\mathbb{P}\left[\left|\eta_{\delta}(X)\right| \leq \bar{\eta}\left(\delta^{-1}\right)\right]=1 .
$$

The converge of the previous ratio to 1 is therefore uniformly controlled by $\bar{\eta}\left(\delta^{-1}\right)$.

Regularity condition. Since the distribution of $X$ is absolutely continuous with respect to the Lebesgue measure and since $B_{0} \in \mathcal{B}$ is a full rank matrix, the random variable $B_{0}^{\top} X$ is also absolutely continuous with a probability distribution function $f_{B_{0}^{\top} X}(\cdot)$ such that $f_{B_{0}^{\top} X}\left(B_{0}^{\top} x\right)>0$ for all $x \in \operatorname{supp}(X)$. The following regularity condition on $f_{B_{0}^{\top} X}(\cdot)$ is required. For all $(s, t) \in$ $(\operatorname{supp}(X))^{2}$, there exists a constant $c_{0}>0$ such that

$$
\left|f_{B_{0}^{\top} X}\left(B_{0}^{\top} s\right)-f_{B_{0}^{\top} X}\left(B_{0}^{\top} t\right)\right| \leq c_{0}\left\|B_{0}^{\top}(s-t)\right\|_{\infty},
$$

where $\|\cdot\|$ denotes any norm on $\mathbb{R}^{q}$.

We can now state the main result of this section. The following notations are used. For all positive definite $q \times q$ matrix $M$ and $s \in \mathbb{R}^{q}$, let $D(s, M):=\left\{t \in \mathbb{R}^{q} ;\left\|M^{-1}(s-t)\right\|_{\infty} \leq 1\right\}$ be the ball of center $s$ and radius $M$. For $v \geq 1$ and $s \in \mathbb{R}$, let $\tilde{L}_{s}(v):=\int_{1}^{v} u^{s-1} \ln u d u$. Finally, for all random variable $W$, let $\delta_{\alpha}(W=\cdot)$ be the measurable function defined for $t \in \operatorname{supp}(W)$ and $\alpha \in(0,1)$ by

$$
\delta_{\alpha}(W=t):= \begin{cases}Q(0 \mid W=t)-Q(\alpha \mid W=t) & \text { if } Q(0 \mid W=t)<\infty \\ 1 / Q(\alpha \mid W=t) & \text { if } Q(0 \mid W=t)=\infty\end{cases}
$$


Theorem 2. Assume that there exists a full rank matrix $B_{0} \in \mathcal{B}$ such that $\mathcal{S}\left(B_{0}\right)$ is a TDR subspace and suppose conditions (9) and (10) hold. Let $\left(\alpha_{n}\right),\left(\beta_{n}\right)$ and $\left(H_{n}\right)$ be sequences such that $\alpha_{n} \rightarrow 0, \beta_{n} / \alpha_{n} \rightarrow 0, n\left|H_{n}\right| \alpha_{n} \rightarrow \infty, \tau_{n} \ln ^{2}\left(\alpha_{n} / \beta_{n}\right) \rightarrow 0$ and $\tau_{n}^{-1}\left\|H_{n}\right\|_{\infty} \rightarrow 0$, where $\tau_{n}:=\left(n\left|H_{n}\right| \alpha_{n}\right)^{-1 / 2}\left[\ln \left(n\left|H_{n}\right| \alpha_{n}\right)\right]^{1 / 2}$. If there exists $\xi \in(0,1)$ such that for all $x \in \operatorname{supp}(X)$,

$$
\max \left\{\sup _{(t, \zeta) \in A_{n}}\left|\frac{\mathbb{P}\left(Y>Q\left(\zeta \mid B_{0}^{\top} X=B_{0}^{\top} x\right) \mid B_{0}^{\top} X=t\right)}{\zeta}-1\right| ; \bar{\eta}\left(\xi \delta_{\alpha_{n}}^{-1}(X=x)\right)\right\}=\mathcal{O}\left(\tau_{n}\right),
$$

where $A_{n}:=D\left(B_{0}^{\top} x, H_{n}\right) \times\left[\xi \nu \alpha_{n}, \xi^{-1} \alpha_{n}\right]$, if $\tau_{n}^{-1} \operatorname{ERV}\left(\alpha_{n}, u \mid X=x\right) \rightarrow 0$ locally uniformly on $u \in(0, \infty)$ and

$$
\lim _{n \rightarrow \infty} \tau_{n}^{-1} \max \left\{\left|\frac{a\left(\alpha_{n}^{-1} \mid x\right)}{Q\left(\alpha_{n} \mid X=x\right)}-\gamma_{+}(x)\right| ; \frac{\operatorname{ERV}\left(\alpha_{n}, \beta_{n} / \alpha_{n} \mid X=x\right)}{\tilde{L}_{\gamma(x)}\left(\alpha_{n} / \beta_{n}\right)}\right\}=0,
$$

then, there exists a Borel set $\mathcal{A} \in \mathcal{B}\left(\mathbb{R}^{p}\right)$ with $\mathbb{P}(X \in \mathcal{A})=1$ and such that for all $x \in \mathcal{A}$,

$$
\frac{\check{Q}_{n}\left(\beta_{n} \mid B_{0}, x\right)-Q\left(\beta_{n} \mid X=x\right)}{a\left(\alpha_{n}^{-1} \mid X=x\right) \tilde{L}_{\gamma(x)}\left(\alpha_{n} / \beta_{n}\right)}=\mathcal{O}_{\mathbb{P}}\left(\tau_{n}\right) .
$$

Remarks.

- Convergence of the conditional quantile estimator cannot be obtained for every $x \in \operatorname{supp}(X)$ since the TCI property is defined only almost surely.

- In (12), two conditions are gathered:

The first one is a classical regularity condition of the function $\mathbb{P}\left(Y>y \mid B_{0}^{\top} X=\cdot\right)$ for large values of $y$. This condition is essential in a conditional framework (see for instance Daouia et al. [7] and Gardes [16]). A careful reading of the proof shows that this condition only needs to be satisfied for $x \in \mathcal{A}$. This condition involves the conditional distribution of $Y$ given $B_{0}^{\top} X$. In Lemma 3 (see Appendix A), it is shown that it can be replaced by a regularity condition on the conditional distribution of $Y$ given $X$ which is often more convenient to check. Note also that the parameter $\nu$ in the set $A_{n}$ is the one used in the estimators (7) and (8) of $\gamma(x)$ and $a\left(\alpha_{n}^{-1} \mid x\right)$.

The second condition is required to deal with the TDR subspace. Roughly speaking, this condition ensures that the ratio $\mathbb{P}\left(Y>\mathcal{Y}_{\delta}\left(B_{0}^{\top} X\right) \mid X\right) / \mathbb{P}\left(Y>\mathcal{Y}_{\delta}\left(B_{0}^{\top} X\right) \mid B_{0}^{\top} X\right)$ converges to 1 as $\delta \rightarrow 0$ sufficiently fast in order to obtain a consistent estimator of $Q\left(\alpha_{n} \mid X=x\right)$ with rate of convergence $\tau_{n}$.

- We would like now to show that if $q<p$ (i.e. if the dimension of the covariate can be reduced), the new estimator $\check{Q}_{n}\left(\beta_{n} \mid B_{0}, x\right)$ is more efficient than the estimator $\check{Q}_{n}\left(\beta_{n} \mid x\right):=\check{Q}_{n}\left(\beta_{n} \mid I_{p}, x\right)$ which does not take into account the existence of a TDR subspace. Assume that conditions of Theorem 2 are satisfied for sequences $\left(\alpha_{n}\right),\left(\beta_{n}\right)$ and a bandwidth matrix $H_{n}=h_{n} I_{q}$ where $\left(h_{n}\right)$ is a positive sequence tending to 0 . Let $M_{n}:=h_{n}^{q / p} I_{p}$. If there exists $\xi \in(0,1)$ such that

$$
\sup _{(t, \zeta) \in \tilde{A}_{n}}\left|\frac{\mathbb{P}(Y>Q(\zeta \mid X=x) \mid X=t)}{\zeta}-1\right|=\mathcal{O}\left(\tau_{n}\right)
$$

where $\tilde{A}_{n}:=D\left(x, M_{n}\right) \times\left[\xi \alpha_{n}, \xi^{-1} \alpha_{n}\right]$ and if $\tau_{n} h_{n}^{q / p} \rightarrow 0$ with $\tau_{n}:=\left[n h_{n}^{q} \alpha_{n} / \ln \left(n h_{n}^{q} \alpha_{n}\right)\right]^{-1 / 2}$, then, $\check{Q}_{n}\left(\beta_{n} \mid x\right)$ computed with the bandwidth matrix $M_{n}$ and $\check{Q}_{n}\left(\beta_{n} \mid B_{0}, x\right)$ computed with $H_{n}$ are both consistent estimators of $Q\left(\alpha_{n} \mid X=x\right)$ with the same rate of convergence $\tau_{n}$. Since $h_{n} / h_{n}^{q / p} \rightarrow 0$ (when $q<p$ ), estimator $\check{Q}_{n}\left(\beta_{n} \mid B_{0}, x\right)$ will perform better in practice because, to keep the same rate of convergence, estimator $\check{Q}_{n}\left(\beta_{n} \mid x\right)$ has to use observations located far away from the target leading to an important bias. To illustrate this fact, let $B_{0}=(1,2)^{\top} / \sqrt{5}$ and $x_{0}=(1 / 2,1 / 2)^{\top}$. Suppose 
that $\mathcal{S}\left(B_{0}\right)$ is a TDR subspace and that one is interested in the estimation of a conditional extreme quantile of $Y$ given $X=x_{0}$ for a random vector $X=\left(X^{(1)}, X^{(2)}\right)^{\top}$ with $X^{(1)}$ and $X^{(2)}$ independent and uniformly distributed on $(0,1)$. In this situation, the points of interest are those located on the set $\left\{x=\left(x^{(1)}, x^{(2)}\right) ; B_{0}^{\top} x=B_{0}^{\top} x_{0}\right\}$. On Figure 1, observations among $n=500$ independent copies $X_{1}, \ldots, X_{n}$ of $X$ for which $K\left(h_{n}^{-1} B_{0}^{\top}\left(x_{0}-X_{i}\right)\right)>0$ (left panel) and $K\left(M_{n}^{-1}\left(x_{0}-X_{i}\right)\right)>0$ (right panel) are represented for $h_{n}=1 / 10$. It clearly appears that the observations used to compute $\check{Q}_{n}\left(\cdot \mid B_{0}, x\right)$ (right panel) are more relevant than the ones used in $\check{Q}_{n}(\cdot \mid x)$ (left panel).

\subsection{Conditional extreme quantile estimation: the case $B_{0}$ unknown}

First, a procedure to estimate the subspace $\mathcal{S}\left(B_{0}\right)$ (i.e. the matrix $B_{0}$ ) is proposed. In what follows, it is assumed that $\operatorname{supp}(X)=\mathbb{R}^{p}$. In this case, we have the following result.

Proposition 3. If $\operatorname{supp}(X)=\mathbb{R}^{p}$ then a minimum TDR subspace is unique.

The starting point for the estimation of $B_{0}$ is a result showing that $B_{0}$ can be seen as the solution of a minimization problem. Let us first introduce some notation. For $J \in \mathbb{N}^{*}$ and for any matrix $B \in \mathcal{B}$, let $\left\{\Pi_{j}\left(B^{\top} X\right), j=1, \ldots, J\right\}$ be a random partition of $\operatorname{supp}(X)$. In the sequel, it is assumed that for all $B \in \mathcal{B}$ and all $j=1, \ldots, J, \mathbb{P}\left(X \in \Pi_{j}\left(B^{\top} X\right) \mid B^{\top} X\right)>0$ a.s. (an example of such a partition is given in Section 4). Let us also introduce the function $T:(0,1) \times \mathcal{B} \mapsto[0, \infty]$ defined by

$$
T(\alpha, B):=\sum_{j=1}^{J}\left\{\mathbb{E}\left[\frac{\mathbb{P}\left(\left\{Y>Q\left(\alpha \mid B^{\top} X\right)\right\} \cap\left\{X \in \Pi_{j}\left(B^{\top} X\right)\right\} \mid B^{\top} X\right)}{\alpha \mathbb{P}\left(X \in \Pi_{j}\left(B^{\top} X\right) \mid B^{\top} X\right)}\right]-1\right\}^{2} .
$$

According to the second statement of Theorem 1 , the quantity $T\left(\alpha, B_{0}\right)$ is close to 0 for small values of $\alpha$. This argument suggests that an approximation of $B_{0}$ can be obtained by minimizing the function $T(\alpha, B)$ with $\alpha$ small. More precisely, introducing the notation

$$
\tilde{B}_{0}(\alpha):=\underset{B \in \mathcal{B}}{\arg \min } T(\alpha, B),
$$

we have the following result.

Theorem 3. Assume that there exists a full rank matrix $B_{0} \in \mathcal{B}$ such that $\mathcal{S}\left(B_{0}\right)$ is a minimum TDR subspace and that $\operatorname{supp}(X)=\mathbb{R}^{p}$. If for all $\kappa>0$ and $\varepsilon>0$, there exists $\alpha_{0} \in(0,1)$ such that for all $\alpha \in\left(0, \alpha_{0}\right), \mathbb{P}\left[\delta_{\alpha}\left(B_{0}^{\top} X\right)<\kappa\right]=1$ and

$$
\sup _{B \in \mathcal{B}}\left|\min \left\{\left|\frac{\mathbb{P}\left(Y>Q\left(\alpha \mid B^{\top} X\right) \mid X\right)}{\alpha}-1\right| ;\left|\frac{\mathbb{P}\left(Y>Q\left(\alpha \mid B^{\top} X\right) \mid X\right)}{\alpha}-1\right|^{-1}\right\}-\tilde{L}(B)\right| \leq \varepsilon,
$$

for some function $\tilde{L}(\cdot): \mathcal{B} \mapsto[0, \infty)$ then $\left\|\tilde{B}_{0}(\alpha)-B_{0}\right\| \rightarrow 0$ as $\alpha \rightarrow 0$ where $\|\cdot\|$ is any matrix norm.

The condition on the random variable $\delta_{\alpha}\left(B_{0}^{T} X\right)$ (which was defined in (11)) entails that $Q\left(\alpha \mid B_{0}^{\top} X=\right.$ $\left.B_{0}^{\top} x\right)$ converges to the endpoint $Q\left(0 \mid B_{0}^{\top} X=B_{0}^{\top} x\right)$ uniformly on $x \in \operatorname{supp}(X)$ as $\alpha$ goes to 0 . Condition (15) ensures that, uniformly on $B \in \mathcal{B},\left|\alpha^{-1} \mathbb{P}\left(Y>Q\left(\alpha \mid B^{\top} X\right) \mid X\right)-1\right|$ admits a positive (possibly infinite) limit as $\alpha$ goes to 0 .

As a conclusion, Proposition 3 and Theorem 3 ensure that the solution of the minimization problem (14) converges to the unique direction $B_{0}$ as $\alpha \rightarrow 0$. This naturally motivates us to estimate $B_{0}$ by replacing $T(\alpha, B)$ in (14) by a reasonable estimator with $\alpha$ sufficiently small. Note that a 
similar procedure was used by Ichimura [25] to estimate the direction of a single-index model.

To construct this estimator, let us introduce a sequence $\left(\alpha_{n}\right)$ converging to 0 with the sample size. The sample analog of $T\left(\alpha_{n}, B\right)$ is given by:

$$
\frac{1}{n^{2}} \sum_{j=1}^{J}\left\{\sum_{i=1}^{n}\left(\frac{\Phi_{n, j}\left(B^{\top} X_{i}\right)}{\alpha_{n} p_{j}\left(B^{\top} X_{i}\right)}-1\right)\right\}^{2},
$$

with for $B \in \mathcal{B}, z \in \operatorname{supp}\left(B^{\top} X\right)$ and $j \in\{1, \ldots, J\}, p_{j}(z):=\mathbb{P}\left(X \in \Pi_{j}\left(B^{\top} X\right) \mid B^{\top} X=z\right) f_{B^{\top} X}(z)$ (where $f_{B^{\top} X}(\cdot)$ is the probability density function of $B^{\top} X$ ) and

$$
\frac{\Phi_{n, j}(z)}{f_{B^{\top} X}(z)}:=\mathbb{P}\left(\left\{Y>Q\left(\alpha \mid B^{\top} X=z\right)\right\} \cap\left\{X \in \Pi_{j}\left(B^{\top} X\right)\right\} \mid B^{\top} X=z\right) .
$$

Obviously, in practice, random variables $\Phi_{n, j}\left(B^{\top} X_{i}\right)$ and $p_{j}\left(B^{\top} X_{i}\right)$ are not observed and must be replaced by their respective kernel estimators:

$$
\widehat{\Phi}_{n, j}\left(B^{\top} X_{i}\right):=\sum_{\ell \neq i} \mathbb{I}_{\left\{Y_{\ell}>\widehat{Q}_{n,-i}\left(\alpha_{n} \mid B^{\top} X_{i}\right)\right\}} \mathbb{I}_{\left\{X_{\ell} \in \Pi_{j}\left(B^{\top} X_{i}\right)\right\}} K\left(H_{n}^{-1} B^{\top}\left(X_{i}-X_{\ell}\right)\right),
$$

where $\widehat{Q}_{n,-i}\left(\alpha_{n} \mid B^{\top} X_{i}\right)$ is the conditional quantile estimator defined in (4) computed without the couple $\left(X_{i}, Y_{i}\right)$ and

$$
\widehat{p}_{j}\left(B^{\top} X_{i}\right):=\sum_{\ell \neq i} \mathbb{I}_{\left\{X_{\ell} \in \Pi_{j}\left(B^{\top} X_{i}\right)\right\}} K\left(H_{n}^{-1} B^{\top}\left(X_{i}-X_{\ell}\right)\right) .
$$

We can now introduce our estimator of $B_{0}$ :

$$
\widehat{B}_{0, n}=\widehat{B}_{0, n}\left(H_{n}, \alpha_{n}\right):=\underset{B \in \mathcal{B}}{\arg \min } \widehat{T}_{n}(B),
$$

where $\widehat{T}_{n}(B)$ is obtained by replacing in (16) the unobserved random variables $\Phi_{n, j}\left(B^{\top} X_{i}\right)$ and $p_{j}\left(B^{\top} X_{i}\right)$ by $\widehat{\Phi}_{n, j}\left(B^{\top} X_{i}\right)$ and $\widehat{p}_{j}\left(B^{\top} X_{i}\right)$.

We can now propose a more useful estimator of $Q\left(\beta_{n} \mid X=x\right)$ than the one obtained in Section 3.1. Replacing $B_{0}$ by $\widehat{B}_{0, n}$ in (6) leads to the estimator $\check{Q}_{n}\left(\beta_{n} \mid \widehat{B}_{0, n}, x\right)$ than can be use in the more realistic situation where the true direction $B_{0}$ is unknown.

The simulation study presented in Section 4 seems to prove that this estimation procedure does work in practice. The optimization problem (17) is solved by using a coordinate search method (see Section 4.1 for more details). Note that when $q>1$, the computational cost of this solving method is important. This is the reason why we focus only on the situation $q=1$ in the simulation study.

Of course, one may wonder if the obtained statistic $\widehat{B}_{0, n}$ is a good estimator (in some sense) of $B_{0}$. To establish the theoretical consistency of $\widehat{B}_{0, n}$, a possible way is to follow the lines of the proof of [25, Theorem 5.1] where an $M$-estimator of the direction in a single-index model is proposed. This proof requires the uniform consistency on $B \in \mathcal{B}$ of the estimator $\widehat{T}_{n}(B)$ of $T(B)$ to be shown. This is an interesting but non-trivial result that is beyond the scope of the present paper.

\section{Simulation study}

\subsection{Estimation of the TDR subspace in practice}

Let $(X, Y)$ be a random vector and assume that there exists a full rank matrix $B_{0}$ of rank $q<p$ such that $\mathcal{S}\left(B_{0}\right)$ is a TDR subspace. Starting from $n$ independent copies $\left(X_{1}, Y_{1}\right), \ldots,\left(X_{n}, Y_{n}\right)$ 
of the random couple $(X, Y)$, we present below a procedure to compute the estimator (17) of the direction $B_{0}$.

Construction of the partition - Let $D=\left[d_{1}, \ldots, d_{p}\right]$ be a $p \times p$ orthogonal matrix such that $\mathcal{S}(B)$ is spanned by $\left\{d_{1}, \ldots, d_{q}\right\}$. The matrix $D$ is obtained by using the Gram-Schmidt process. Let $m\left(B^{\top} x\right)$ be the conditional marginal median of $X$ given $B^{\top} X=B^{\top} x$ and for $\ell \in\{1, \ldots, p-q\}$, let us introduce the half spaces

$$
\begin{aligned}
& E_{\ell}\left(B^{\top} x\right):=\left\{s \in \mathbb{R}^{p} ; d_{\ell+q}^{\top} s>d_{\ell+q}^{\top} m\left(B^{\top} x\right)\right\} \\
& \text { and } \quad \bar{E}_{\ell}\left(B^{\top} x\right):=\left\{s \in \mathbb{R}^{p} ; d_{\ell+q}^{\top} s \leq d_{\ell+q}^{\top} m\left(B^{\top} x\right)\right\} \text {. }
\end{aligned}
$$

An element of the partition $\left\{\Pi_{j}\left(B^{\top} X=B^{\top} x\right), j=1, \ldots, J\right\}$ is the intersection of $p-q$ half spaces. More specifically, an element of the partition is a set $E_{1}^{*} \cap \ldots \cap E_{p-q}^{*}$ where for $\ell \in\{1, \ldots, p-q\}$, $E_{\ell}^{*} \in\left\{E_{\ell}\left(B^{\top} x\right), \bar{E}_{\ell}\left(B^{\top} x\right)\right\}$. There is thus $J=2^{p-q}$ elements in the partition. Obviously, if $\operatorname{supp}(X)=\mathbb{R}^{p}$, then, for all $x \in \mathbb{R}^{p}$ and, for all $j \in\{1, \ldots, J\}, \mathbb{P}\left(X \in \Pi_{j}\left(B^{\top} X\right) \mid B^{\top} X=B^{\top} x\right)>0$. In practice, since the conditional marginal median $m\left(B^{\top} x\right)$ is unknown, it is replaced by its empirical estimator $\widehat{m}_{n}\left(B^{\top} x\right):=\left(\widehat{m}_{n, j}\left(B^{\top} x\right), j=1, \ldots, J\right)^{\top}$ where for $j=1, \ldots, J, \widehat{m}_{n, j}\left(B^{\top} x\right)$ is the empirical median of the $\mathrm{j}$-th component of the observations falling in $D\left(B^{\top} x, H_{n}\right)$. This choice of random partition ensures that, for all $x \in \operatorname{supp}(X)$, the number of available observations in each element of $D\left(B^{\top} x, H_{n}\right) \cap \Pi_{j}\left(B^{\top} X=B^{\top} x\right)$ is approximatively the same. For a better view of the previously constructed partition, let us give an example. Let $X=\left(X^{(1)}, X^{(2)}\right)^{\top}$ be a random vector where $X^{(1)}$ and $X^{(2)}$ are independent and uniformly distributed on $[0,1]$. In Figure 2, the partition $\left\{\Pi_{1}\left(B^{\top} X=B^{\top} x\right), \Pi_{2}\left(B^{\top} X=B^{\top} x\right)\right\}$ of $\operatorname{supp}(X)=[0,1]^{2}$ is represented for $B=(1,2)^{\top} / \sqrt{5}$ and $x=(3 / 5,1 / 5)^{\top}$. Using $n=500$ independent copies of $X$ and taking $H_{n}=50 / n=0.1$, the conditional marginal median $m\left(B^{\top} x\right)$ is estimated by $\widehat{m}_{n}\left(B^{\top} x\right)=(0.416,0.280)^{\top}$.

Computation of $\widehat{T}_{n}(B)$ - The previously defined partition is used to compute the sample analog (16) of $T\left(\alpha_{n}, B\right)$ for any given matrix $B$. Using the whole sample $\left\{X_{1}, \ldots, X_{n}\right\}$ is time consuming since the partition must be computed for each observations $X_{i}, i=1, \ldots, n$. We thus choose to use only a random subsample of size $n_{0}:=\lfloor a n\rfloor$ for a given $a \in(0,1)$ to compute $(16)$. Extensive simulation studies show that this procedure does not affect too much the quality of the obtained estimator of $B_{0}$ (see Section 4.3).

Minimization of the function $\widehat{T}_{n}(\cdot)$ - To solve this optimization problem, we choose to use the coordinate search method (see for instance [23]). Starting with a matrix $B_{0} \in \mathcal{B}$, this method tries to find a new matrix $B_{1} \in \mathcal{B}$ such that the value of the objective function at $B_{1}$ is smaller than the one at $B_{0}$. The new matrix $B_{1}$ is obtained in the following way: we first compute a matrix $\check{B}_{1}$ by adding or subtracting the search distance $\delta$ to a single coordinate in each row of $B_{0}$. We then compute the matrix $B_{1} \in \mathcal{B}$ such that $\operatorname{span}\left(B_{1}\right)=\operatorname{span}\left(\check{B}_{1}\right)$. If such a matrix can be found then $B_{0}$ is replaced by $B_{1}$, the search distance $\delta$ is increased and the previous procedure is repeated. If not, the previous procedure is repeated with $B_{0}$ and a smaller value of $\delta$. More specifically, the coordinate search method used in this paper is described below:

- Initialization: Take $B^{(0)} \in \mathcal{B}, \delta^{(0)}>0$ and $\delta^{\text {tol }}>0$.

- Step $k$ : let $\left(e_{1}, \ldots, e_{p}\right)$ be the canonical basis of $\mathbb{R}^{p}$ and, for $i=1, \ldots, 2 p$, let $\tilde{e}_{i}:=(-1)^{i} e_{\lceil i / 2\rceil}$. For $\left(i_{1}, \ldots, i_{q}\right) \in\{1, \ldots, 2 p\}^{q}$, compute the matrix $\tilde{B}_{\left(i_{1}, \ldots, i_{q}\right)} \in \mathcal{B}$ whose columns are the first $q$ 
linearly independent columns of the projection matrix on the set spanned by the columns of the matrix $B^{(k-1)}+\delta^{(k-1)} \tilde{E}$ with $\tilde{E}=\left[\tilde{e}_{i_{1}}, \ldots, \tilde{e}_{i_{q}}\right]$. Denoting by $\mathcal{B}^{(k)}$ the set of the $(2 p)^{q}$ matrices $\tilde{B}_{\left(i_{1}, \ldots, i_{q}\right)},\left(i_{1}, \ldots, i_{q}\right) \in\{1, \ldots, 2 p\}^{q}$, there are two possibilities

- if $\widehat{T}_{n}\left(B^{(k-1)}\right) \leq \min \left\{\widehat{T}_{n}(B), B \in \mathcal{B}^{(k)}\right\}$, then

$$
B^{(k)}=B^{(k-1)} \text { and } \delta^{(k)}=\delta^{(k-1)} / 2,
$$

- if $\widehat{T}_{n}\left(B^{(k-1)}\right)>\min \left\{\widehat{T}_{n}(B), B \in \mathcal{B}^{(k)}\right\}$, then

$$
B^{(k)}=\underset{B \in \mathcal{B}(k)}{\arg \min } \widehat{T}_{n}(B) \text { and } \delta^{(k)}=2 \delta^{(k-1)} .
$$

- If $\delta^{(k)}>\delta^{\text {tol }}$, go to step $k+1$, else, the algorithm is stopped.

The next section is dedicated to the presentation of the models considered in the simulation study.

\subsection{Model setting}

In what follows, the $p$ components of the random vector $X$ are independent and distributed as a Gaussian random variable with mean $1 / 2$ and variance $\sigma^{2}$. The dimension of the explanatory variable $X$ is fixed to $p=4$ and the response $Y$ is generated according to the following models: for positive functions $g_{0}(\cdot), g_{1}(\cdot), g_{2}(\cdot)$, for $B_{0} \in \mathbb{R}^{4}$ and $B_{1} \in \mathbb{R}^{4}$, the conditional quantile of $Y$ given $X=x$ is given for $\alpha \in(0,1)$ and $x \in \mathbb{R}^{p}$ by:

$$
\begin{aligned}
& \text { Model } 1-Q_{1}(\alpha \mid X=x):=[\ln (1 /(1-\alpha))]^{-g_{0}\left(B_{0}^{\top} x\right)}\left[1+g_{1}\left(B_{1}^{\top} x\right) \exp \left(-\alpha^{-1}\right)\right]^{-1} . \\
& \text { Model } 2-Q_{2}(\alpha \mid X=x):=g_{2}\left(B_{0}^{\top} x\right)-[\ln (1 /(1-\alpha))]^{g_{0}\left(B_{0}^{\top} x\right)}\left[1+g_{1}\left(B_{1}^{\top} x\right) \exp \left(-\alpha^{-1}\right)\right] . \\
& \text { Model } 3-Q_{3}(\alpha \mid X=x):=[\ln (1 / \alpha)]^{g_{0}\left(B_{0}^{\top} x\right)}\left[1+g_{1}\left(B_{1}^{\top} x\right) \exp \left(-\alpha^{-1}\right)\right]^{-1} .
\end{aligned}
$$

Denoting by $S_{1}(y \mid x)$ (resp. $\left.S_{2}(y \mid x), S_{3}(y \mid x)\right)$ the conditional survival function $\mathbb{P}(Y>y \mid X=x)$ when $(X, Y)$ is distributed from Model 1 (resp. Model 2, Model 3), it is proved in Lemma 4 that for all $x \in \mathbb{R}^{p}$ and $\delta>0$

$$
\begin{aligned}
S_{1}\left(\delta^{-1} \mid x\right) & =\delta^{1 / g_{0}\left(B_{0}^{\top} x\right)} L_{1}\left(\delta^{-1} \mid x\right), S_{2}\left(g_{2}\left(B_{0}^{\top} x\right)-\delta \mid x\right)=S_{1}\left(\delta^{-1} \mid x\right) \\
\text { and } \quad S_{3}\left(\delta^{-1} \mid x\right) & =\exp \left[-\delta^{-1 / g_{0}\left(B_{0}^{\top} x\right)} L_{3}\left(\delta^{-1} \mid x\right)\right],
\end{aligned}
$$

where $L_{1}(y \mid x)$ and $L_{3}(y \mid x)$ converge to 1 as $y \rightarrow \infty$.

The first statement of [22, Theorem 1.2.1] entails that $S_{1}(\cdot \mid x)$ is in the maximum domain of attraction of Fréchet with positive extreme value index $\gamma(x)=g_{0}\left(B_{0}^{\top} x\right)$ and, as a consequence, condition (5) is satisfied by $Q_{1}(\cdot \mid X=x)$.

According to the second statement of [22, Theorem 1.2.1], $S_{2}(\cdot \mid x)$ is in the maximum domain of attraction of Weibull with a negative extreme value index $\gamma(x)=-g_{0}\left(B_{0}^{\top} x\right)$ and a right endpoint $Q_{2}(0 \mid X=x)=g_{2}\left(B_{0}^{\top} x\right)$.

Finally, for all $x \in \mathbb{R}^{p}, S_{3}(\cdot \mid x)$ is a Weibull-tail distribution (see for instance [19] for a definition). As a consequence, $S_{3}(\cdot \mid x)$ belongs to the Gumbel maximum domain of attraction and thus $Q_{3}(\cdot \mid X=x)$ satisfies (5) with an extreme value index equal to 0 .

The following parameterization is considered in the sequel: $g_{0}(\cdot):=\tilde{g}(z ; 1 / 3,8 / 3)$ and $g_{2}(\cdot):=$ $\tilde{g}(z ; 1,10)$ where for $a<b$ and $z \in \mathbb{R}$,

$$
\tilde{g}(z ; a, b)=a \mathbb{I}_{(-\infty, 0)}(z)+\left(a+b \frac{\exp (2 z)-1}{\exp (6 / \sqrt{5})-1}\right) \mathbb{I}_{[0,3 / \sqrt{5})}(z)+(a+b) \mathbb{I}_{[3 / \sqrt{5}, \infty)}(z) .
$$


Note that for all $z \in \mathbb{R}, \tilde{g}(z ; a, b) \in[a, a+b]$. The function $g_{1}(\cdot)$ is defined by $g_{1}(z)=\mathbb{I}_{(-\infty, 0)}(z)+$ $\exp (5 z) \mathbb{I}_{[0,2)}(z)+\exp (10) \mathbb{I}_{[2, \infty)}(z)$. Finally, $B_{0}^{\top}:=(2,1,0,0) / \sqrt{5}$ and $B_{1}^{\top}:=(0,0,1,1)$. It can be shown (see Lemma 4 , appendix A) that for all these models, $\mathcal{S}\left(B_{0}\right)$ is a TDR subspace (with $q=1$ ) and that condition (9) is satisfied.

\subsection{TDR subspace and conditional quantile estimation}

For each model introduced in Section 4.2, $N=100$ samples of size $n=4000$ are generated. Our purpose is to appreciate the finite sample performance of the TDR subspace estimator defined in (17) and to compare three different estimators of the conditional extreme quantile $Q\left(\beta_{n} \mid X=x\right)$ of order $\beta_{n}=2 / n$ : (a) estimator $\check{Q}_{n}\left(\beta_{n} \mid B_{0}, x\right)$ for the (unrealistic) situation where the TDR direction $B_{0}$ is known, (b) the more useful plug-in estimator $\check{Q}_{n}\left(\beta_{n} \mid \widehat{B}_{0, n}, x\right)$ and (c) the classical estimator $\check{Q}_{n}\left(\beta_{n} \mid x\right):=\check{Q}_{n}\left(\beta_{n} \mid I_{p}, x\right)$ when the existence of a TDR subspace is not taken into account.

TDR subspace estimator To compute the estimator, we take $\alpha_{n}=n^{-1 / 3}, H_{n}=n^{-2 / 9}$ and a subsample of size $n_{0}=100$. Obviously, hidden parameters $\alpha_{n}$ and $H_{n}$ have a strong influence on the quality of the estimation. In the real data application, a procedure to choose these parameters is proposed. In the simulation study, we decide to fix the values of $\alpha_{n}$ and $H_{n}$ by eye in order to focus only on the estimation of the TDR subspace. The influence of the subsample size $n_{0}$ is less critical. To support this assertion, the estimation of $B_{0}$ has been considered under model 1 with $\sigma=1 / 5$ for $n_{0} \in\{2,4, \ldots, 20,25,50,75,100,150,200,250,300\}$. In figure 3 , the empirical quantiles of order $0.05,0.5$ and 0.95 of $\left\|\widehat{B}_{0, n}-B_{0}\right\|_{F}$ (where for all matrix $B,\|B\|_{F}:=\left[\operatorname{tr}\left(B^{\top} B\right)\right]^{1 / 2}$ is the Frobenius norm) are represented as a function of $n_{0}$. It appears that for $n_{0}>75$, the quality of the estimation does not clearly depends on $n_{0}$. Recall also that an initialization is required in the coordinate search method used to compute the TDR subspace estimator. In this simulation study, the initial vector $B^{(0)}$ is randomly chosen. More specifically, $B^{(0)}=\left(u_{1}, \ldots, u_{p}\right) /\left[u_{1}^{2}+\ldots+\right.$ $\left.u_{p}^{2}\right]^{1 / 2}$ where $u_{1}, \ldots, u_{p}$ are realizations of $p$ independent random variables uniformly distributed on $[-1,1]$.

The estimator $\widehat{B}_{0, n}$ of $B_{0}$ is compared with the one obtained by the SIR method. Recall that the SIR estimator $\widehat{B}_{0, n}^{\mathrm{SIR}}$ corresponds to the $q$ eigenvectors associated to the $q$ largest eigenvalues of the matrix $\widehat{\Sigma}_{n}^{-1} \widehat{\Gamma}_{n}$ with

$$
\begin{aligned}
\widehat{\Sigma}_{n} & :=\frac{1}{n} \sum_{i=1}^{n}\left(X_{i}-\frac{1}{n} \sum_{i=1}^{n} X_{i}\right)\left(X_{i}-\frac{1}{n} \sum_{i=1}^{n} X_{i}\right)^{\top} \\
\text { and } \widehat{\Gamma}_{n}: & : \sum_{j=1}^{J} \frac{n_{j}}{n}\left(\frac{1}{n_{j}} \sum_{i: Y_{i} \in R_{j}} X_{i}-\frac{1}{n} \sum_{i=1}^{n} X_{i}\right)\left(\frac{1}{n_{j}} \sum_{i: Y_{i} \in R_{j}} X_{i}-\frac{1}{n} \sum_{i=1}^{n} X_{i}\right)^{\top},
\end{aligned}
$$

where $\left\{R_{1}, \ldots, R_{J}\right\}$ are non-overlapping slices that cover the range of $Y$ and $n_{j}$ is the number of $Y_{i}$ 's lying in $R_{j}$. To compute the SIR estimator, the R package dr (see [36]) was used with the default method to construct the slices. The SIR method focus only on the central part of the conditional distribution of $Y$ given $X$ and is thus generally not adapted for the estimation of the TDR subspace. The accuracy of the TDR direction estimators is measured by the Frobenius distance to the true direction $B_{0}$. 
Conditional quantile estimators Here also, since our purpose is to compare three different estimators of the same quantity, it seems more adequate to fix the hyperparameters $\alpha_{n}$ and $H_{n}$. As before, we take $\alpha_{n}=n^{-1 / 3}$. For estimators $\check{Q}_{n}\left(\beta_{n} \mid B_{0}, x\right)$ and $\check{Q}_{n}\left(\beta_{n} \mid \widehat{B}_{0, n}, x\right)$, the bandwidth is $H_{n}=n^{-2 / 9}$. For $\check{Q}_{n}\left(\beta_{n} \mid x\right)$, the $p \times p$ matrix $H_{n}$ is given by $\left[n^{-2 / 9}\right]^{1 / 4} I_{4}$. According to the third remark after Theorem 2, this choice ensures that estimators $\check{Q}_{n}\left(\beta_{n} \mid B_{0}, x\right)$ and $\check{Q}_{n}\left(\beta_{n} \mid x\right)$ share the same rate of convergence. To compute the conditional tail index estimator (7) and the auxiliary function estimator (8) we take $\nu=0.02$ and $\varphi(\cdot)=\ln (1 / \cdot)$ (this choice was suggested in [16]). Concerning the kernel function $K(\cdot)$ we take the Epanechnikov kernel. It is well known that in practice, the choice of the kernel function is not crucial (see for instance [10]). To assess the estimation procedure, we compute for each replication the error

$$
\mathrm{E}_{Q}(\check{B}):=\frac{1}{\operatorname{card}(\mathcal{L})} \sum_{x \in \mathcal{L}}\left(\ln \frac{\check{Q}\left(\beta_{n} \mid \check{B}, x\right)}{Q\left(\beta_{n} \mid X=x\right)}\right)^{2},
$$

where $\mathcal{L}$ is the set $\left\{\left(x_{1}, \ldots, x_{4}\right) ; x_{i} \in\{1 / 4,1 / 2,3 / 4\}\right.$ for $\left.i=1, \ldots, 4\right\}$ with $\operatorname{card}(\mathcal{L})=3^{4}=81$ and $\check{B} \in\left\{\widehat{B}_{0, n}, \widehat{B}_{0, n}^{\mathrm{SIR}}, I_{p}\right\}$.

Results are gathered in Table 1 . As expected, the conditional extreme quantile estimator $\check{Q}_{n}\left(\beta_{n} \mid B_{0}, \cdot\right)$ is the best among the three considered statistics. Unfortunately, this estimator can only be used in the ideal situation where $B_{0}$ is known. Nevertheless, the plug-in estimator $\check{Q}_{n}\left(\beta_{n} \mid \hat{B}_{0, n}, \cdot\right)$ provides similar results and outperforms in each case the classical estimator $\check{Q}_{n}\left(\beta_{n} \mid I_{p}, \cdot\right)$ (for which the existence of a TDR subspace is not assumed). One can remark that even for a large sample size $(n=4000)$, the classical estimator performs very poorly for a 4 -dimensional covariate. There is thus a real benefit in using our dimension reduction procedure. Note also that the TDR estimator $\hat{B}_{0, n}$ provides good estimation of the true direction $B_{0}$ while, as expected, the SIR estimator $\hat{B}_{0, n}^{\mathrm{SIR}}$ is not able to find the true direction. It appears in fact that $\hat{B}_{0, n}^{\mathrm{SIR}}$ is often close to the "central" direction $B_{1}=(0,0,1,1)^{\top}$. For instance, when $\sigma=1 / 3,\left\|\hat{B}_{0, n}^{\mathrm{SIR}}-B_{1}\right\|_{F}=0.016$ for Model 1, $\left\|\hat{B}_{0, n}^{\mathrm{SIR}}-B_{1}\right\|_{F}=0.066$ for Model 2 and $\left\|\hat{B}_{0, n}^{\mathrm{SIR}}-B_{1}\right\|_{F}=0.110$ for Model 3. This confirms the fact that classical dimension reduction methods are not adapted to the study of the tail of the conditional distribution. Note also that the value of the standard deviation $\sigma$ has an influence on the estimation of $B_{0}$. Except for Model 2, the performance of $\hat{B}_{0, n}$ deteriorates as $\sigma$ decreases. One possible explanation is that when $\sigma$ is small, the variance of $\tilde{\gamma}\left(B_{0}^{\top} X\right)$ is also small is thus the true direction is difficult to capture. Finally, to illustrate the sensitivity to the hidden parameters $\alpha_{n}$ and $H_{n}$, I estimate the TDR direction and the conditional quantile of order $\beta_{n}=2 / n$ under model 1 with $\sigma=1 / 8$ and with the following hidden parameters: (1) $\alpha_{n}=H_{n}=n^{-1 / 3}$, (2) $\alpha_{n}=n^{-1 / 3}$ and $H_{n}=n^{-1 / 10}$ and (3) $\alpha_{n}=H_{n}=n^{-1 / 10}$. Results are given in Table 2 . As expected, the conditional quantile estimator is sensitive to a bad choice of $\alpha_{n}$ and $H_{n}$. The estimator of $B_{0}$ seems to be more robust.

Choice of the TDR dimension In this simulation study, we assume that the true dimension $q$ of the minimum TDR subspace is known. Obviously, in practice, this is not the case and $q$ need to be estimated. In a non extreme-value context, some approaches for the estimation of $q$ can be found in the literature (see for instance [6] where a general permutation test is proposed and [5] for the use of information criterion). Adaptation of these methods to our situation is beyond the scope of this paper. Nevertheless, in our setting, a possible way to check that the choice $q=1$ is reasonable is to look at the scatter plot of the largest observations $\left\{Y_{n-\lfloor n \alpha\rfloor+1, n} \leq \ldots \leq Y_{n, n}\right\}$ versus the projections $\left\{\widehat{B}_{0, n}^{\top} X_{i}^{*}, i=1, \ldots,\lfloor n \alpha\rfloor\right\}$ where $X_{i}^{*}$ is the covariate associated to the order 
statistic $Y_{n-i+1, n}$ and $\widehat{B}_{0, n} \in \mathbb{R}^{p}$ is the estimated direction obtained in Section 4.1. The presence of a relationship between extreme values of $Y$ and their corresponding projections on $\widehat{B}_{0, n}$ is a good indicator that the projection $\widehat{B}_{0, n}^{\top} X$ contains most of the information on the tail distribution of $Y$. This scatter plot is represented on Figure 4 for a sample of size $n=4000$ generated according to Model 1 with $\sigma=1 / 3$. As before, $\alpha_{n}=n^{-1 / 3}$ and $H_{n}=n^{-2 / 9}$. On the left panel, the direction $B_{0}$ is estimated by the procedure described in Section 4.1 while on the right panel, this direction is estimated by the SIR approach. A strong link between extreme values of $Y$ and their corresponding projections can be seen on the left panel. In contrast, extreme values seem uniformly distributed on the right panel. This scatter plot is therefore a useful graphical tool that permits us to visually check the relevance of a TDR model with $q=1$. The right panel confirms again that classical dimension reduction methods are not always useful when one is interested in the tail of the conditional distribution.

\section{Data analysis}

According to the world health organization, atmospheric pollutants may cause serious effects on public health and on environment. One of the most dangerous pollutant in urban areas is ozone. It is generated in the air when others pollutants (called primary pollutants) react with atmospheric oxygen and weather conditions (temperature, humidity, etc.).

Our objective is to understand the relationship existing between the concentration of some primary pollutants and the daily maximum surface concentration of ozone. Our study is based on data collected in Chicago from 1987 to 2000 during $n=4841$ days. These data are available on the $\mathrm{R}$ package NMMAPS Data Lite. The dataset consists in daily concentrations of different pollutants such as ozone $\left(\mathrm{O}_{3}\right)$, particular matter with diameter smaller than 10 microns or 25 microns $\left(\mathrm{PM}_{10}\right.$ or $\left.\mathrm{PM}_{25}\right)$, sulphur dioxide $\left(\mathrm{SO}_{2}\right)$, nitrogen dioxide $\left(\mathrm{NO}_{2}\right)$, carbon monoxide $(\mathrm{CO})$, etc. Various meteorology and mortality variables were also recorded. These data were considered by many authors in a dimension reduction framework (see for instance [33] and [38]).

More specifically, let $Y$ be the concentration of $\mathrm{O}_{3}$ (in parts per billion) and $X$ be the covariate vector of dimension $p=4$ corresponding to the daily maximum concentrations of $\mathrm{PM}_{10}, \mathrm{SO}_{2}, \mathrm{NO}_{2}$ and CO. Note that for numerical convenience, the components of $X$ are centered and normalized. We are interested in the estimation of extreme quantiles of order $\beta_{n}=1 / n$ of the conditional distribution of $Y$ given $X=x$ with two different possible scenarios for $x$. Let $x_{\tau}^{\mathrm{PM}_{10}}, x_{\tau}^{\mathrm{SO}_{2}}, x_{\tau}^{\mathrm{NO}_{2}}$ and $x_{\tau}^{\mathrm{CO}}$ be the empirical quantile of order $1-\tau \in(0,1)$ of the (centered and normalized) daily maximum values of $\mathrm{PM}_{10}, \mathrm{SO}_{2}, \mathrm{NO}_{2}$ and $\mathrm{CO}$. The first scenario is $x=\left(x_{.5}^{\mathrm{PM}_{10}}, x_{.5}^{\mathrm{SO}_{2}}, x_{.5}^{\mathrm{NO}_{2}}, x_{.5}^{\mathrm{CO}}\right)^{\top}$. This joint vector is quite close to a situation observed in Chicago during the period 1987-2000 with moderate values of the four primary pollutants. A second likely scenario is $x=\left(x_{.5}^{\mathrm{PM}_{10}}, x_{.25}^{\mathrm{SO}_{2}}, x_{.05}^{\mathrm{NO}_{2}}, x_{.05}^{\mathrm{CO}}\right)^{\top}$ corresponding to large values for $\mathrm{NO}_{2}$ and $\mathrm{CO}$.

In a first step, we assume the existence of a TDR subspace for $Y$ given $X$ of dimension $q=1$. To compute the TDR estimator proposed in this paper, we use the Epanechnikov kernel. A choice for the bandwidth $H_{n}$, the percentage of largest observations $\alpha_{n}$ and the size $n_{0}$ of the random subsample is also required. As mentioned in Section 4.2, the choice of $n_{0}$ is not critical and we fix it to $n_{0}=100$. The choice of $H_{n}$ and $\alpha_{n}$ is more important and the following data-driven procedure 
is used. Let $\mathcal{H}:=\{0.05, \ldots, 0.25\}$ and $\mathcal{A}:=\{0.01, \ldots, 0.1\}$ be two sets of possible 10 equi-spaced values for $H_{n}$ and $\alpha_{n}$. The selected values of $H_{n}$ and $\alpha_{n}$ are given by

$$
\left(\hat{H}_{n}, \hat{\alpha}_{n}\right):=\underset{(h, \alpha) \in \mathcal{H} \times \mathcal{A}}{\arg \min } \widehat{T}_{n}\left(\widehat{B}_{0, n}(h, \alpha)\right) .
$$

The idea motivating this criteria is that for well chosen $H_{n}$ and $\alpha_{n}$, the value of $\widehat{T}_{n}\left(\widehat{B}_{0, n}\left(H_{n}, \alpha_{n}\right)\right)$ must be close to 0 . For the Chicago air pollution dataset, this procedure leads to $\hat{h}_{n}=0.094$ and $\hat{\alpha}_{n}=0.09\left(\right.$ with $\left.\widehat{T}_{n}\left(\widehat{B}_{0, n}(0.094,0.09)\right)=0.006\right)$. The estimated direction is

$$
\widehat{B}_{0, n}=(0.175,-0.036,0.962,-0.207)^{\top} .
$$

Note that the coordinate search method was initialized with different vectors $B^{(0)}$, all these initialization leading to very similar values of $\widehat{B}_{0, n}$.

In the first step, the existence of a TDR subspace of dimension $q=1$ was assumed. We must now check if this assumption seems reasonable for the Chicago air pollution dataset. The scatter plot introduced in Section 4.3 (Choice of the TDR dimension) is presented on Figure 5. A pattern clearly appears in this plot. This is a first clue that the projection of $X$ on $\hat{B}_{0, n}$ contains a non negligible amount of information on the tail distribution of $Y$. The scatter plot can be completed by the value of the correlation between $Y$ and $\hat{B}_{0, n}^{\top} X$. This correlation is equal to $\operatorname{Cor}\left(Y, \hat{B}_{0, n}^{\top} X\right)=0.58$ and has to be compared to correlations between $Y$ and each individual covariate: $\operatorname{Cor}\left(Y, P M_{10}\right)=0.37 ; \operatorname{Cor}\left(Y, S O_{2}\right)=0.05 ; \operatorname{Cor}\left(Y, N O_{2}\right)=0.55$ and $\operatorname{Cor}(Y, C O)=0.17$. It appears that $Y$ is more correlated with the projection of $X$ on $\hat{B}_{0, n}$ than with each individual covariate. We can also remark that $\operatorname{Cor}\left(Y, N O_{2}\right) \approx \operatorname{Cor}\left(Y, \hat{B}_{0, n}^{\top} X\right)$ which is not surprising since $\widehat{B}_{0, n}$ is close to the direction $b_{0}:=(0,0,1,0)^{\top}\left(\left\|\hat{B}_{0, n}-b_{0}\right\|_{F}=0.08\right)$. The covariate $\mathrm{NO}_{2}$ seems to bring the most important information on large values of ozone concentration.

We can now proceed to the estimation of conditional extreme quantiles for the two considered scenarios. In order to have an information on the distribution of the conditional quantile estimator, a jackknife resampling method is used. More specifically, we first select the observations corresponding to the $\left\lfloor n \hat{\alpha}_{n}\right\rfloor=435$ largest values of $Y$. A sample distribution of the conditional quantile estimator is then obtained by leaving out each selected observations and computing the estimator. For each scenario, we represent the box-plot of the jackknife distribution (see Figure 6). It appears that the worst scenario for large values of ozone concentration is the second one: very important ozone concentration is more likely to be observed when concentrations of $\mathrm{NO}_{2}$ and $\mathrm{CO}$ are important. Controlling levels of $\mathrm{NO}_{2}$ and $\mathrm{CO}$ can thus lead to a reduction in ozone concentration. This conclusion is in line with the paper of Han et al. [24] where it is shown that "the levels of $\mathrm{O}_{3}$ and $\mathrm{NO}_{2}$ are inextricably linked".

\section{Conclusion}

We propose in this paper a new dimension reduction framework dedicated to the study of the tail of a distribution in presence of a covariate of dimension $p$. An estimation procedure of the reduced subspace and conditional extreme quantiles is presented. It appears on our simulation study that even for a moderate dimension $p$ of the covariate (for instance $p=4$ ), the classical kernel estimator of conditional extreme quantiles fails to approximate the true quantile while our procedure provides significantly better results. 


\section{References}

[1] Araújo Santos, P., Fraga Alves, M.I. and Gomes, M.I. (2006). Peaks Over Random Threshold methodology for tail index and high quantile estimation, REVSTAT, 4(3), 227-247.

[2] Basu, D. and Pereira, C.A.B. (1983). Conditional independence in statistics, Sankhyā, 45(3), Series A, 324-337.

[3] Chaudhuri, P. (1991). Nonparametric estimates of regression quantiles and their local Bahadur representation, Annals of Statistics, 19(2), 760-777.

[4] Cook, R.D. (1994). On the interpretation of regression plots, Journal of the American Statistical Association, 89(425), 177-189.

[5] Cook, R.D. and Forzani, L. (2009). Likelihood-based sufficient dimension reduction, Journal of the American Statistical Association, 104, 197-208.

[6] Cook, R.D. and Weisberg, S. (1991). Discussion of "Sliced Inverse Regression for dimension reduction", Journal of the American Statistical Association, 86, 328-332.

[7] Daouia, A., Gardes, L. and Girard, S. (2013). On kernel smoothing for extremal quantile regression, Bernoulli, 19(5B), 2557-2589.

[8] Daouia, A., Gardes, L., Girard, S. and Lekina, A. (2011). Kernel estimators of extreme level curves, Test, 20(2), 311-333.

[9] Davison, A.C. and Smith, R.L. (1990). Models for exceedances over high thresholds, Journal of the Royal Statistical Society, series B, 52, 393-442.

[10] Davison, A.C. and Ramesh, N.I. (2000). Local likelihood smoothing of sample extremes, Journal of the Royal Statistical Society, series B, 62, 191-208.

[11] Dekkers, A. L. M., Einmahl, J. H. J. and de Haan, L. (1989). A moment estimator for the index of an extreme-value distribution, The Annals of Statistics, 17(4), 1833-1855.

[12] Eastoe, E.F. and Tawn, J.A. (2009). Modelling non-stationary extremes with application to surface level ozone, Journal of the Royal Statistical Society, series C, 58, 25-45.

[13] Fisher, R. A. and Tippett, L. H. C. (1928). Limiting forms of the frequency distribution of the largest or smallest member of a sample, Proceedings of the Cambridge Philosophical Society, 24, 180-190.

[14] Fraga Alves, M. I., Gomes, M. I., de Haan, L. and Neves, C. (2007). A note on second order conditions in extreme value theory: linking general and heavy tail conditions, REVSTAT Statistical Journal, 5(3), 285-304.

[15] Gannoun, A., Girard, S., Guinot, C. and Saracco, J. (2004). Sliced inverse regression in reference curves estimation, Computational Statistics and Data Analysis, 46(1), 103-122.

[16] Gardes, L. (2015). A general estimator for the extreme value index: applications to conditional and heteroscedastic extremes, Extremes, 18(3), 479-510.

[17] Gardes, L. and Girard, S. (2010). Conditional extremes from heavy-tailed distributions: an application to the estimation of extreme rainfall return levels, Extremes, 13(2), 177-204. 
[18] Gardes, L. and Girard, S. (2012). Functional kernel estimators of large conditional quantiles, Electronic Journal of Statistics, 6, 1715-1744.

[19] Gardes, L. and Girard, S. (2016). On the estimation of the functional Weibull tail-coefficient, Journal of Multivariate Analysis, 146, 29-45

[20] Gasser, T. and Müller, H.G. (1984). Estimating regression functions and their derivatives by the kernel method, Scandinavian Journal of Statistics, 11, 171-185.

[21] Gnedenko, B. V. (1943). Sur la distribution limite du terme maximum d'une série aléatoire, Annals of Mathematics, 44, 423-453.

[22] de Haan, L. and Ferreira, A. (2006). Extreme Value Theory: An Introduction, Springer.

[23] Hooke, R. and Jeeves, T.A. (1961). Direct search solution of numerical and statistical problems, Journal of the ACM, 8(2), 212-229.

[24] Han, S., Bian, H., Feng, Y., Liu, A., Li, ., Zeng, F. and Zhang, X. (2011). Analysis of the relationship between $\mathrm{O}_{3}, \mathrm{NO}$ and $\mathrm{NO}_{2}$ in Tianjin, China, Aerosol and Air Quality Research, 11, 128-139.

[25] Ichimura, H. (1993). Semiparametric least squares (SLS) and weighted SLS estimation of single-index models, Journal of econometrics, 58, 71-120.

[26] Koenker, R. and Basset, G.S. (1978). Regression quantiles, Econometrica, 46, 33-50.

[27] Li, K.C. (1991). Sliced inverse regression for dimension reduction. Journal of the American Statistical Association, 86, 316-327.

[28] Li, K.C. (1992). On principal hessian directions for data visualization and dimension reduction: another application of Stein's lemma, Journal of the American Statistical Association, 87, $1025-1039$.

[29] Nadaraya, E.A. (1964). On estimating regression, Theory of Probability and its Application, $\mathbf{9}(\mathbf{1}), 141-142$.

[30] Northrop, P.J. and Jonathan, P. (2011). Threshold modelling of spatially dependent nonstationary extremes with application to hurricane-induced wave heights, Environmetrics, 22(7), 799-809.

[31] Parzen, E. (1962). On the estimation of a probability density function and mode, Annals of Mathematical Statistics, 33, 1065-1076.

[32] Russell, B.T., Cooley, D., Porter, W.C., Reich, B.J. and Heald C.L. (2016). Data mining for extreme behavior with application to ground level ozone, Annals of Applied Statistics, 10, 1673-1698.

[33] Scrucca, L. (2011). Model-based SIR for dimension reduction, Computational Statistics and Data Analysis, 55, 3010-3026.

[34] Wang, E. and Tsai, C. (2009). Tail Index Regression, Journal of the American Statistical Association, 104(487), 1233-1240. 
[35] Watson, G. S. (1964). Smooth regression analysis, Sankhyā, 26(15), 175-184.

[36] Weisberg, W. (2002). Dimension reduction regression in R, Journal of Statistical Software, 7, $1-22$.

[37] Wu, T.Z., Yu, K. and Yu, Y. (2010). Single-index quantile regression, Journal of Multivariate Analysis, 101, 1607-1621.

[38] Xia, Y. (2009). Model checking in regression via dimension reduction, Biometrika, 96(1), $133-148$.

[39] Yu, K. and Jones, M.C. (1998). Local Linear Quantile Regression, Journal of the American Statistical Association, 93(441), 228-237.

\section{Appendix - Proofs}

\section{Preliminary results}

The first Lemma is a probability result that will be helpful in the proof Theorem 1 .

Lemma 1. Let $(\Omega, \mathcal{F}, \mathbb{P})$ be a probability space. Let $U$ be a positive random variable and $V$ be a $\mathbb{R}^{q}$-valued random vector $(U$ and $V$ are both defined on $(\Omega, \mathcal{F}, \mathbb{P})$ ). Denoting by $\sigma(U)$ (resp. $\sigma(V)$ ) the $\sigma$-algebra generated by $U$ (resp. $V$ ), it is assumed that $\sigma(V) \subset \sigma(U)$. If there exists $\theta \geq 1$ such that for all $F \in \sigma(U)$,

$$
\int_{F} \mathbb{E}(U \mid V) d \mathbb{P} \leq \theta \int_{F} U d \mathbb{P}\left(\text { or } \int_{F} \mathbb{E}(U \mid V) d \mathbb{P} \geq \theta^{-1} \int_{F} U d \mathbb{P}\right),
$$

then $\mathbb{E}(U \mid V) \leq \theta U$ a.s. (or $\mathbb{E}(U \mid V) \geq \theta^{-1} U$ a.s.).

Proof - Assume that for all $F \in \sigma(U)$

$$
\int_{F} \mathbb{E}(U \mid V) d \mathbb{P} \leq \theta \int_{F} U d \mathbb{P}
$$

(the proof for the other case is similar). First, we suppose that $U$ is a positive simple function. More specifically, let $\left\{A_{1}, \ldots, A_{k}\right\}$ be $k$ disjoint elements of $\mathcal{F}$ with $0<\mathbb{P}\left(A_{i}\right)<1$ for all $i \in\{1, \ldots, k\}$. We assume that

$$
U=\sum_{i=1}^{k} c_{i} \mathbb{I}_{A_{i}}+c_{k+1} \mathbb{I}_{\left(A_{1} \cup \ldots \cup A_{k}\right)^{\mathrm{C}}},
$$

where for all $A \subset \Omega, A^{\mathrm{C}}$ is the complement of $A$ in $\Omega$ and $c_{i}>0$ for all $i \in\{1, \ldots, k\}$. Since $\sigma(V) \subset \sigma(U)$, one can assume without loss of generality that $\sigma(V)=\sigma\left(\left\{A_{1}, \ldots, A_{\ell}\right\}\right)$ with $1 \leq$ $\ell<k$ (the situation where $\ell=k$, i.e. $\sigma(U)=\sigma(V)$ is trivial). It is then easy to check that

$$
\mathbb{E}(U \mid V)=\sum_{i=1}^{\ell} c_{i} \mathbb{I}_{A_{i}}+\xi \mathbb{I}_{\left(A_{1} \cup \ldots \cup A_{\ell}\right)^{\mathrm{C}}}
$$

where

$$
\xi:=\sum_{i=\ell+1}^{k} c_{i} \frac{\mathbb{P}\left(A_{i}\right)}{\mathbb{P}\left[\left(A_{1} \cup \ldots \cup A_{\ell}\right)^{\mathrm{C}}\right]}+c_{k+1} \frac{\mathbb{P}\left[\left(A_{1} \cup \ldots \cup A_{k}\right)^{\mathrm{C}}\right]}{\mathbb{P}\left[\left(A_{1} \cup \ldots \cup A_{\ell}\right)^{\mathrm{C}}\right]}
$$


We thus have to show that for every $i \in\{\ell+1, \ldots, k\}, \xi \leq \theta c_{i}$ and that, if $\mathbb{P}\left[\left(A_{1} \cup \ldots \cup A_{k}\right)^{\mathrm{C}}\right] \neq 0$, $\xi \leq \theta c_{k+1}$. From (18), for all $F \in \sigma(U)=\sigma\left(\left\{A_{1}, \ldots, A_{k}\right\}\right)$,

$$
\sum_{i=1}^{\ell} c_{i} \mathbb{P}\left(A_{i} \cap F\right)+\xi \mathbb{P}\left[\left(A_{1} \cup \ldots \cup A_{\ell}\right)^{\mathrm{C}} \cap F\right] \leq \theta \sum_{i=1}^{k} c_{i} \mathbb{P}\left(A_{i} \cap F\right)+c_{k+1} \mathbb{P}\left[\left(A_{1} \cup \ldots \cup A_{k}\right)^{\mathrm{C}} \cap F\right] .
$$

For $j \in\{\ell+1, \ldots, k\}$, taking $F=A_{j}$ in the previous inequality leads to $\xi \leq \theta c_{j}$ since $\mathbb{P}\left(A_{j}\right)>0$. Furthermore, taking $F=\left(A_{1} \cup \ldots \cup A_{k}\right)^{\mathrm{C}}$ entails that $\xi \mathbb{P}\left[\left(A_{1} \cup \ldots \cup A_{k}\right)^{\mathrm{C}}\right] \leq \theta \mathbb{P}\left[\left(A_{1} \cup \ldots \cup A_{k}\right)^{\mathrm{C}}\right]$. The result is thus proved for all positive simple functions. Since any positive measurable function is the pointwise limit of an increasing sequence of positive simple function, we conclude the proof by using the Lebesgue's monotone convergence theorem.

The next lemma is a technical result used in the proof of Theorem 4 .

Lemma 2. Assume that there exists a full rank $p \times q$ matrix $B_{0}$ such that $\mathcal{S}\left(B_{0}\right)$ is a TDR subspace for $Y$ given $X$ and that condition (5) holds. For $x \in \operatorname{supp}(X)$, let $\left(y_{n}(u \mid x)\right)$ be a sequence such that $\left[y_{n}(u \mid x)-Q\left(u \alpha_{n} \mid X=x\right)\right] / a\left(\alpha_{n}^{-1} \mid X=x\right) \rightarrow 0$ locally uniformly on $u \in(0, \infty)$. The following statements hold almost everywhere for $x \in \operatorname{supp}(X)$ :

(i) Let $\nu \in(0,1)$. For all $u \in[\nu, 1], y_{n}(u \mid x)=Q\left(\beta \mid B_{0}^{\top} X=B_{0}^{\top} x\right)$ with $\beta \in\left[\xi \nu \alpha_{n}, \xi^{-1} \alpha_{n}\right]$ for $\xi<1$ as near as you like to 1 .

(ii) Let $u \in(0,1)$ and $\xi<1$ as near as we like to 1 . If $Q(0 \mid X=x)<\infty$ then $Q(0 \mid X=$ $x)-y_{n}(u \mid x) \leq \xi^{-1} \delta_{\alpha_{n}}(X=x)$ and if $Q(0 \mid X=x)=+\infty$ then $\left[y_{n}(u \mid x)\right]^{-1} \leq \xi^{-1} \delta_{\alpha_{n}}(X=x)$.

Proof - We start by proving the first statement. From (5), one has for $x \in \operatorname{supp}(X)$ that $\left[y_{n}(u \mid x)-Q\left(\alpha_{n} \mid X=x\right)\right] / a\left(\alpha_{n}^{-1} \mid X=x\right)-L_{\gamma(x)}(1 / u) \rightarrow 0$ locally uniformly on $u \in(0, \infty)$. Now, since $\mathcal{S}\left(B_{0}\right)$ is a TDR subspace, [22, Lemma 1.2.12] entails that $\left[Q\left(\alpha_{n} \mid X=x\right)-Q\left(\alpha_{n} \mid B_{0}^{\top} X=\right.\right.$ $\left.\left.B_{0}^{\top} x\right)\right] / a\left(\alpha_{n}^{-1} \mid X=x\right)$ converges to 0 almost everywhere for $x \in \operatorname{supp}(X)$. As a first conclusion,

$$
y_{n}(u \mid x)=Q\left(\alpha_{n} \mid B_{0}^{\top} X=B_{0}^{\top} x\right)+a\left(\alpha_{n}^{-1} \mid x\right)\left[L_{\gamma(x)}(1 / u)+o(1)\right],
$$

locally uniformly on $u \in(0, \infty)$ and almost everywhere for $x \in \operatorname{supp}(X)$. From Lemma 1, the distribution function of $Y$ given $B_{0}^{\top} X$ belongs to a maximum domain of attraction with an auxiliary function equivalent to $a(\cdot \mid x)$. Thus, according to [22, Theorem 1.1.6], one can find $\xi<1$ as close as we like to 1 such that for all $u \in[\nu, 1], \mathbb{P}\left(Y>y_{n}(u \mid x) \mid B_{0}^{\top} X=B_{0}^{\top} x\right) \geq \nu \xi \alpha_{n}$. Hence,

$$
\left.y_{n}(u \mid x)=Q\left(\mathbb{P}\left(Y>y_{n}(u \mid x) \mid B_{0}^{\top} X=B_{0}^{\top} x\right)\right) \mid B_{0}^{\top} X=B_{0}^{\top} x\right) \leq Q\left(\nu \xi \alpha_{n} \mid B_{0}^{\top} X=B_{0}^{\top} x\right) .
$$

Mimicking the proof of (19), we show that $y_{n}(u \mid x) \geq Q\left(\xi^{-1} \alpha_{n} \mid B_{0}^{\top} X=B_{0}^{\top} x\right)$ and thus conclude the proof of the first statement.

Let us now focus on the second statement. Assume first that $Q(0 \mid X=x)<\infty$ (this implies that $\gamma(x) \leq 0)$. Using the first statement with $B_{0}=I_{p}$, one has for all $u \leq 1$ and for $\tilde{\xi}$ as near as we like to 1 that $Q(0 \mid X=x)-y_{n}(u \mid x) \leq Q(0 \mid X=x)-Q\left(\tilde{\xi}^{-1} \alpha_{n} \mid X=x\right)$. Now from [22, Lemma 1.2.9], one has that $\delta_{\alpha_{n}}^{-1}(X=x) a\left(\alpha_{n}^{-1} \mid X=x\right) \rightarrow-\gamma(x)$ as $n$ goes to infinity. Hence, from (5), one can find $\xi<1$ as near as we want to 1 such that

$$
\frac{Q(0 \mid X=x)-y_{n}(u \mid x)}{\delta_{\alpha_{n}}(X=x)} \leq 1+\frac{Q\left(\alpha_{n} \mid X=x\right)-Q\left(\tilde{\xi}^{-1} \alpha_{n} \mid X=x\right)}{\delta_{\alpha_{n}}(X=x)} \leq \xi^{-1} .
$$

Now, suppose that $Q(0 \mid X=x)=+\infty$ (and thus that $\gamma(x) \geq 0)$. Applying again the first statement with $B_{0}=I_{p}$, one has for all $u \leq 1$ that $y_{n}(u \mid x) \geq Q\left(\tilde{\xi}^{-1} \alpha_{n} \mid X=x\right)$ where $\tilde{\xi}<1$ is as close as we 
want to 1 . Since $\delta_{\alpha_{n}}(X=x) a\left(\alpha_{n}^{-1} \mid X=x\right)=a\left(\alpha_{n}^{-1} \mid X=x\right) / Q\left(\alpha_{n} \mid X=x\right) \rightarrow \gamma(x)$ as $n$ goes to infinity, condition (5) entails that $\delta_{\alpha_{n}}(X=x) Q\left(\tilde{\xi}^{-1} \alpha_{n} \mid X=x\right)=1+\delta_{\alpha_{n}}(X=x)\left[Q\left(\tilde{\xi}^{-1} \alpha_{n} \mid X=\right.\right.$ $\left.x)-Q\left(\alpha_{n} \mid X=x\right)\right] \rightarrow \tilde{\xi}^{\gamma(x)}$. Hence, one can find $\xi<1$ as close as we want to 1 and such that for $n$ large enough, $y_{n}(u \mid x) \geq \xi \delta_{\alpha_{n}}^{-1}(X=x)$ and the proof is complete.

\section{Proofs of main results}

Proof of Theorem $1-$ First, we prove that $(i)$ implies (ii). Let $\varepsilon>0$. There exists $\kappa>0$ such that for all $\delta \in(0, \kappa]$,

$$
\Delta_{\delta}(X, Z):=\left|\frac{\mathbb{P}\left(Y>\mathcal{Y}_{\delta}(Z) \mid X, Z\right)}{\mathbb{P}\left(Y>\mathcal{Y}_{\delta}(Z) \mid Z\right)}-1\right| \leq \varepsilon
$$

Remarking that for all non-zero bounded and positive function $h(\cdot)$,

$$
\mathbb{E}\left(\mathbb{I}_{\left\{Y>\mathcal{Y}_{\delta}(Z)\right\}} h(X) \mid Z\right)=\mathbb{E}\left[h(X) \mathbb{E}\left(\mathbb{I}_{\left\{Y>\mathcal{Y}_{\delta}(Z)\right\}} \mid X, Z\right) \mid Z\right] .
$$

it is easy to check that, almost surely,

$$
\left|\frac{\mathbb{E}\left(\mathbb{I}_{\left\{Y>\mathcal{Y}_{\delta}(Z)\right\}} h(X) \mid Z\right)}{\mathbb{P}\left(Y>\mathcal{Y}_{\delta}(Z) \mid Z\right) \mathbb{E}(h(X) \mid Z)}-1\right| \leq \mathbb{E}\left[\left|\frac{\mathbb{P}\left(Y>\mathcal{Y}_{\delta}(Z) \mid X, Z\right)}{\mathbb{P}\left(Y>\mathcal{Y}_{\delta}(Z) \mid Z\right)}-1\right| \frac{h(X)}{\mathbb{E}(h(X) \mid Z)} \mid Z\right] \leq \varepsilon .
$$

Let us now show that $(i i)$ implies $(i)$. We thus assume that for all $\varepsilon>0$, there exist $\kappa>0$ such that for all $\delta \in(0, \kappa]$, inequality (20) holds. Let $A \in \mathcal{B}\left(\mathbb{R}^{p}\right)$ and $B \in \mathcal{B}\left(\mathbb{R}^{q}\right)$, we have

$$
\begin{aligned}
& \int_{\{X \in A\} \cap\{Z \in B\}} \mathbb{P}\left(Y>\mathcal{Y}_{\delta}(Z) \mid Z\right) d \mathbb{P}=\mathbb{E}\left\{\mathbb{E}\left[\mathbb{I}_{\{X \in A\}} \mathbb{E}\left(\mathbb{I}_{\left\{Y>\mathcal{Y}_{\delta}(Z)\right\}} \mathbb{I}_{\{Z \in B\}} \mid Z\right) \mid Z\right]\right\} \\
= & \int_{\{Z \in B\}} \mathbb{P}\left(Y>\mathcal{Y}_{\delta}(Z) \mid Z\right) \mathbb{P}(X \in A \mid Z) d \mathbb{P} \\
= & \int_{\{Z \in B\}} \mathbb{P}\left[\left\{Y>\mathcal{Y}_{\delta}(Z)\right\} \cap\{X \in A\} \mid Z\right] \frac{\mathbb{P}(X \in A \mid Z) \mathbb{P}(X \in A \mid Z)}{\mathbb{P}\left[\left\{Y>\mathcal{Y}_{\delta}(Z)\right\} \cap\{X \in A\} \mid Z\right]} d \mathbb{P} .
\end{aligned}
$$

Let $\varepsilon \in(0,1)$. Applying inequality (20) with $h(X)=\mathbb{I}_{A}(X)$ leads to

$$
\int_{\{X \in A\} \cap\{Z \in B\}} \mathbb{P}\left(Y>\mathcal{Y}_{\delta}(Z) \mid Z\right) d \mathbb{P} \leq \frac{1}{1-\varepsilon} \int_{\{Z \in B\}} \mathbb{P}\left[\left\{Y>\mathcal{Y}_{\delta}(Z)\right\} \cap\{X \in A\} \mid Z\right] d \mathbb{P}
$$

and

$$
\int_{\{X \in A\} \cap\{Z \in B\}} \mathbb{P}\left(Y>\mathcal{Y}_{\delta}(Z) \mid Z\right) d \mathbb{P} \geq \frac{1}{1+\varepsilon} \int_{\{Z \in B\}} \mathbb{P}\left[\left\{Y>\mathcal{Y}_{\delta}(Z)\right\} \cap\{X \in A\} \mid Z\right] d \mathbb{P} .
$$

Since

$$
\int_{\{Z \in B\}} \mathbb{P}\left[\left\{Y>\mathcal{Y}_{\delta}(Z)\right\} \cap\{X \in A\} \mid Z\right] d \mathbb{P}=\int_{\{X \in A\} \cap\{Z \in B\}} \mathbb{P}\left(Y>\mathcal{Y}_{\delta}(Z) \mid X, Z\right) d \mathbb{P},
$$

and by the monotone class theorem we thus have for all $F \in \sigma(X, Z)$ that

$$
\frac{1}{1+\varepsilon} \int_{F} \mathbb{P}\left(Y>\mathcal{Y}_{\delta}(Z) \mid X, Z\right) d \mathbb{P} \leq \int_{F} \mathbb{P}\left(Y>\mathcal{Y}_{\delta}(Z) \mid Z\right) d \mathbb{P} \leq \frac{1}{1-\varepsilon} \int_{F} \mathbb{P}\left(Y>\mathcal{Y}_{\delta}(Z) \mid X, Z\right) d \mathbb{P} .
$$

We conclude the proof by applying Lemma 1 with $U:=\mathbb{P}\left(Y>\mathcal{Y}_{\delta}(Z) \mid X, Z\right)$ and $V=Z$.

Let us now prove that $(i)$ is equivalent to $(i i i)$. Obviously, if $(i)$ holds then (iii) also holds with $s_{\delta}(Z)=\mathbb{P}\left(Y>\mathcal{Y}_{\delta}(Z) \mid Z\right)$ and $\eta_{\delta}(X, Z)=\mathbb{P}\left(Y>\mathcal{Y}_{\delta}(Z) \mid X, Z\right) / \mathbb{P}\left(Y>\mathcal{Y}_{\delta}(Z) \mid Z\right)-1$. 
To show that (iii) implies (i), first remark that the "tower property" of conditional expectations entails that $\mathbb{P}\left(Y>\mathcal{Y}_{\delta}(Z) \mid Z\right)=\mathbb{E}\left[\mathbb{P}\left(Y>\mathcal{Y}_{\delta}(Z) \mid X, Z\right) \mid Z\right]=s_{\delta}(Z)\left[1+\mathbb{E}\left(\eta_{\delta}(X, Z) \mid Z\right)\right]$ a.s. Hence

$$
\frac{\mathbb{P}\left(Y>\mathcal{Y}_{\delta}(Z) \mid X, Z\right)}{\mathbb{P}\left(Y>\mathcal{Y}_{\delta}(Z) \mid Z\right)}=\frac{1+\eta_{\delta}(X, Z)}{1+\mathbb{E}\left(\eta_{\delta}(X, Z) \mid Z\right)} \text { a.s. }
$$

By assumption, for all $\varepsilon \in(0,1)$, there exists $\kappa>0$ such that for all $\delta \in(0, \kappa]$,

$$
\mathbb{P}\left[\left|\frac{\mathbb{P}\left(Y>\mathcal{Y}_{\delta}(Z) \mid X, Z\right)}{\mathbb{P}\left(Y>\mathcal{Y}_{\delta}(Z) \mid Z\right)}-1\right| \leq \frac{2 \varepsilon}{1-\varepsilon}\right]=1
$$

which concludes the proof.

Proof of Proposition 1 - First recall that since $\mathcal{S}\left(B_{0}\right)$ is a TDR subspace, there exists a Borel set $\mathcal{A} \in \mathcal{B}\left(\mathbb{R}^{p}\right)$ with $\mathbb{P}(X \in \mathcal{A})=1$ such that for all $x \in \mathcal{A}, Q(0 \mid X=x)=Q\left(0 \mid B_{0}^{\top} X=B_{0}^{\top} x\right)$. Hence, for all $x \in \mathcal{A},[22$, Lemma 1.2.12] and the definition of the TDR subspace entail that the distribution function $\mathbb{P}\left(Y \leq \cdot B_{0}^{\top} X=B_{0}^{\top} x\right)$ belongs to the maximum domain of attraction of an extreme value distribution with extreme value index $\gamma(x)$ or equivalently that for all $u>0$ and $x \in \mathcal{A}$

$$
\lim _{\alpha \rightarrow 0} \frac{Q\left(u \alpha \mid B_{0}^{\top} X=B_{0}^{\top} x\right)-Q\left(\alpha \mid B_{0}^{\top} X=B_{0}^{\top} x\right)}{a\left(\alpha^{-1} \mid x\right)}=L_{\gamma(x)}(1 / u) .
$$

As a consequence, from [22, Theorem 1.2.6],for all $x \in \mathcal{A}$ there exist positive functions $c\left(\cdot \mid B_{0}^{\top} x\right)$ and $d\left(\cdot \mid B_{0}^{\top} x\right)$ (depending on $x$ only through $\left.B_{0}^{\top} x\right)$ such that for all $y \in\left(y_{0}, Q\left(0 \mid B_{0}^{\top} X=B_{0}^{\top} x\right)\right.$ ),

$$
\mathbb{P}\left(Y>y \mid B_{0}^{\top} X=B_{0}^{\top} x\right)=c\left(y \mid B_{0}^{\top} x\right) \exp \left\{-\int_{y_{0}}^{y} \frac{d s}{d\left(s \mid B_{0}^{\top} x\right)}\right\},
$$

with $c\left(y \mid B_{0}^{\top} x\right) \rightarrow c_{0}>0$ as $y \rightarrow Q\left(0 \mid B_{0}^{\top} X=B_{0}^{\top} x\right)$. Now, let us introduce the differentiable function

$$
S_{0}\left(y \mid B_{0}^{\top} x\right):=\min \left(1, c_{0} \exp \left\{-\int_{y_{0}}^{y} \frac{d s}{d\left(s \mid B_{0}^{\top} x\right)}\right\}\right) .
$$

According to Remarks 1.2.7 and 1.2.8 in [22], the survival function $S_{0}\left(\cdot \mid B_{0}^{\top} x\right)$ belongs to the maximum domain of attraction of an extreme value distribution with extreme value index $\gamma(x)$. More precisely, taking $\tilde{a}\left(\alpha^{-1} \mid B_{0}^{\top} x\right):=d_{0}\left(S_{0}^{\leftarrow}\left(\alpha \mid B_{0}^{\top} x\right)\right)$ where $\left.S_{0}^{\leftarrow}\left(\cdot \mid B_{0}^{\top} x\right)\right)$ is the generalized inverse of $S_{0}^{\leftarrow}\left(\cdot \mid B_{0}^{\top} x\right)$ (recall that for any non-increasing $\Phi(\cdot)$, its generalized inverse is given by $\left.\Phi^{\leftarrow}(\cdot)=\inf \{y ; \Phi(y) \leq \cdot\}\right)$ and where $d_{0}(\cdot):=-S_{0}\left(\cdot \mid B_{0}^{\top} x\right) / S_{0}^{\prime}\left(\cdot \mid B_{0}^{\top} x\right)$, one has for all $u>0$ and $x \in \mathcal{A}$

$$
\lim _{\alpha \rightarrow 0} \frac{S_{0}^{\leftarrow}\left(u \alpha \mid B_{0}^{\top} x\right)-S_{0}^{\leftarrow}\left(\alpha \mid B_{0}^{\top} x\right)}{\tilde{a}\left(\alpha^{-1} \mid B_{0}^{\top} x\right)}=L_{\gamma(x)}(1 / u)
$$

Since the left hand side in the previous limit only depends on $x$ through $B_{0}^{\top} x$, same holds for the right hand side. Hence there exists a real-valued function $\tilde{\gamma}(\cdot)$ such that $\gamma(x)=\tilde{\gamma}\left(B_{0}^{\top} x\right)$ for all $x \in \mathcal{A}$. Finally, since $\mathbb{P}\left(Y>y \mid B_{0}^{\top} X=B_{0}^{\top} x\right) / S_{0}\left(y \mid B_{0}^{\top} x\right) \rightarrow 1$ as $y \rightarrow Q\left(0 \mid B_{0}^{\top} X=B_{0}^{\top} x\right)$, using again [22, Lemma 1.2.12] entails that for all $u>0$ and $x \in \mathcal{A}$

$$
\lim _{\alpha \rightarrow 0} \frac{Q\left(u \alpha \mid B_{0}^{\top} X=B_{0}^{\top} x\right)-Q\left(\alpha \mid B_{0}^{\top} X=B_{0}^{\top} x\right)}{\tilde{a}\left(\alpha^{-1} \mid B_{0}^{\top} x\right)}=L_{\tilde{\gamma}\left(B_{0}^{\top} x\right)}(1 / u) .
$$

We conclude the proof by remarking that (23) and (24) lead to $\tilde{a}\left(\alpha^{-1} \mid B_{0}^{\top} x\right) / a\left(\alpha^{-1} \mid x\right) \rightarrow 1$ as $\alpha$ goes to 0 . 
To prove Theorem 2, the following intermediate result is required. It deals with the asymptotic properties of the conditional survival function estimator given in (3).

Proposition 4. Assume that there exists a full rank matrix $B_{0}$ such that $\mathcal{S}\left(B_{0}\right)$ is a TDR subspace and suppose conditions (9) and (10) hold. Let $\left(\alpha_{n}\right)$ and $\left(H_{n}\right)$ be two sequences such that $\alpha_{n} \rightarrow 0$, $n\left|H_{n}\right| \alpha_{n} \rightarrow \infty$ and $\tau_{n}^{-1}\left\|H_{n}\right\|_{\infty} \rightarrow 0$ with $\tau_{n}:=\left(n\left|H_{n}\right| \alpha_{n}\right)^{-1 / 2}\left[\ln \left(n\left|H_{n}\right| \alpha_{n}\right)\right]^{1 / 2}$. If there exists $\xi \in(0,1)$ such that for all $x \in \operatorname{supp}(X)$,

$$
\max \left\{\sup _{(t, \beta) \in A_{n}}\left|\frac{\mathbb{P}\left(Y>Q\left(\beta \mid B_{0}^{\top} X=B_{0}^{\top} x\right) \mid B_{0}^{\top} X=t\right)}{\beta}-1\right| ; \bar{\eta}\left(\xi \delta_{\alpha_{n}}^{-1}(X=x)\right)\right\}=\mathcal{O}\left(\tau_{n}\right),
$$

where $A_{n}:=D\left(B_{0}^{\top} x, H_{n}\right) \times\left[\xi \nu \alpha_{n}, \xi^{-1} \alpha_{n}\right]$, and if $\tau_{n}^{-1} \operatorname{ERV}\left(\alpha_{n}, u \mid X=x\right) \rightarrow 0$ locally uniformly on $u \in(0, \infty)$ then, for all $\nu \in(0,1)$, there exists a Borel set $\mathcal{A} \in \mathcal{B}\left(\mathbb{R}^{p}\right)$ with $\mathbb{P}(X \in \mathcal{A})=1$ such that for all $x \in \mathcal{A}$ and for all sequences $\left(y_{n}(u \mid x)\right)$ such that $\left[y_{n}(u \mid x)-Q\left(u \alpha_{n} \mid X=x\right)\right] / a\left(\alpha_{n}^{-1} \mid X=\right.$ $x) \rightarrow 0$ locally uniformly on $u \in(0, \infty)$.

$$
\sup _{u \in[\nu, 1]}\left|\frac{\widehat{S}_{n}\left(y_{n}(u \mid x) \mid B_{0}, x\right)}{\mathbb{P}\left(Y>y_{n}(u \mid x) \mid X=x\right)}-1\right|=\mathcal{O}_{\mathbb{P}}\left(\tau_{n}\right) .
$$

Before proving this result, remark that from [16, Lemma 5], Proposition 4 entails that

$$
\frac{Q\left(\alpha_{n} \mid X=x\right)}{a\left(\alpha_{n}^{-1} \mid X=x\right)} \sup _{u \in[\nu, 1]}\left|\frac{\widehat{Q}_{n}\left(u \alpha_{n} \mid B_{0}, x\right)}{Q\left(u \alpha_{n} \mid X=x\right)}-1\right|=\mathcal{O}_{\mathbb{P}}\left(\tau_{n}\right), .
$$

Thus $\widehat{Q}_{n}\left(\cdot \mid B_{0}, x\right)$ is a consistent estimator of $Q(\cdot \mid X=x)$ but under a restrictive condition on the order $\alpha_{n}$ (namely, $n\left|H_{n}\right| \alpha_{n} \rightarrow \infty$ ).

Proof of Proposition 4 - First remark that $\widehat{S}_{n}\left(y_{n}(u \mid x) \mid B_{0}, x\right)=\widehat{\Phi}_{n}(u \mid x) /\left[n\left|H_{n}\right| \widehat{f}_{n, B_{0}^{\top} X}\left(B_{0}^{\top} x\right)\right]$, where

$$
\begin{gathered}
\widehat{f}_{n, B_{0}^{\top} X}\left(B_{0}^{\top} x\right):=\frac{1}{n\left|H_{n}\right|} \sum_{i=1}^{n} K\left(H_{n}^{-1} B_{0}^{\top}\left(x-X_{i}\right)\right) \\
\text { and } \widehat{\Phi}_{n}(u \mid x):=\sum_{i=1}^{n} K\left(H_{n}^{-1} B_{0}^{\top}\left(x-X_{i}\right)\right) \mathbb{I}_{\left\{Y_{i}>y_{n}(u \mid x)\right\}} \text {. }
\end{gathered}
$$

Note that $\widehat{f}_{n, B_{0}^{\top} X}(\cdot)$ is the classical kernel estimator of the probability distribution function $f_{B_{0}^{\top} X}(\cdot)$. Under (10) and since $n\left|H_{n}\right| \rightarrow \infty$, it can be shown (see for instance Parzen [31]) that

$$
\frac{\widehat{f}_{n, B_{0}^{\top} X}\left(B_{0}^{\top} x\right)}{f_{B_{0}^{\top} X}\left(B_{0}^{\top} x\right)}-1=\mathcal{O}_{\mathbb{P}}\left(\left\|H_{n}\right\|_{\infty}\right)+\mathcal{O}_{\mathbb{P}}\left(\left(n\left|H_{n}\right|\right)^{-1 / 2}\right)=o_{\mathbb{P}}\left(\tau_{n}\right)
$$

Let us now focus on the statistics $\widehat{\Phi}_{n}(u \mid x)$. According to [16, Lemma 6], letting $\mu_{n}(u \mid x):=$ $\mathbb{E}\left(\widehat{\Phi}_{n}(u \mid x)\right)$ and $v_{n, x}^{2}:=\ln \left(\mu_{n}(1 \mid x)\right) / \mu_{n}(1 \mid x)$, if the following conditions are satisfied:

(C.1) There exists a positive constant $C_{X}$ such that $K\left(H_{n}^{-1} B_{0}^{\top}\left(x-X_{i}\right)\right) \mathbb{I}_{\left\{Y_{i}>y_{n}(u \mid x)\right\}} \leq C_{X}$ for $n$ large enough and all $u \in[\nu, 1]$,

(C.2) $\mu_{n}(\nu \mid x) \rightarrow \infty$ as $n \rightarrow \infty$ and there exists a positive constant $C_{\mu}$ such that for $n$ large enough $\mu_{n}(\nu \mid x) / \mu_{n}(1 \mid x) \geq C_{\mu}$, 
(C.3)

$$
\sup \left\{\left|\frac{\mu_{n}(u \mid x)}{\mu_{n}\left(u^{\prime} \mid x\right)}-1\right|, u \in[\nu, 1] \text { with }\left|u-u^{\prime}\right| \leq\left(\mu_{n}(1 \mid x)\right)^{-1 / 2}\right\}=\mathcal{O}\left(v_{n, x}\right)
$$

then

$$
\sup _{u \in[\nu, 1]}\left|\frac{\widehat{\Phi}_{n}(u \mid x)}{\mu_{n}(u \mid x)}-1\right|=\mathcal{O}_{\mathbb{P}}\left(v_{n, x}\right) .
$$

Since $K(\cdot)$ is bounded, it is clear that (C.1) holds with $C_{X}=\|K\|_{\infty}$. To check (C.2) and (C.3), the first step is the computation of $\mu_{n}(u \mid x)$. Since $X$ is absolutely continuous with respect to the Lebesgue measure and since $B_{0}$ is a full rank matrix, the random vector $B_{0}^{\top} X$ is also absolutely continuous with support $\operatorname{supp}\left(B_{0}^{\top} X\right)=\left\{B_{0}^{\top} x, x \in \operatorname{supp}(X)\right\}$. Hence,

$$
\mu_{n}(u \mid x)=n \int_{\operatorname{supp}\left(B_{0}^{\top} X\right)} K\left(H_{n}^{-1}\left(B_{0}^{\top} x-t\right)\right) \mathbb{P}\left(Y>y_{n}(u \mid x) \mid B_{0}^{\top} X=t\right) f_{B_{0}^{\top} X}(t) d t .
$$

Letting $s=H_{n}^{-1}\left(B_{0}^{\top} x-t\right)$ leads to

$$
\mu_{n}(u \mid x)=n\left|H_{n}\right| \int_{\mathcal{U}_{q}} K(s) \mathbb{P}\left(Y>y_{n}(u \mid x) \mid B_{0}^{\top} X=B_{0}^{\top} x-H_{n} s\right) f_{B_{0}^{\top} X}\left(B_{0}^{\top} x-H_{n} s\right) d s,
$$

since, for $n$ large enough, $\mathcal{U}_{q} \subset\left\{H_{n}^{-1}\left(B_{0}^{\top} x-t\right), t \in \operatorname{supp}\left(B_{0}^{\top} X\right)\right\}$ where $\mathcal{U}_{q}$ is the unit ball of $\mathbb{R}^{q}$. To sum up, since $f_{B_{0}^{\top} X}\left(B_{0}^{\top} x\right)>0$,

$$
\begin{aligned}
& \frac{\mu_{n}(u \mid x)}{n\left|H_{n}\right| f_{B_{0}^{\top} X}\left(B_{0}^{\top} x\right) \mathbb{P}\left(Y>y_{n}(u \mid x) \mid B_{0}^{\top} X=B_{0}^{\top} x\right)} \\
= & \int_{\mathcal{U}_{q}} K(s) \frac{\mathbb{P}\left(Y>y_{n}(u \mid x) \mid B_{0}^{\top} X=B_{0}^{\top} x-H_{n} s\right)}{\mathbb{P}\left(Y>y_{n}(u \mid x) \mid B_{0}^{\top} X=B_{0}^{\top} x\right)} \frac{f_{B_{0}^{\top} X}\left(B_{0}^{\top} x-H_{n} s\right)}{f_{B_{0}^{\top} X}\left(B_{0}^{\top} x\right)} d s .
\end{aligned}
$$

Remarking that $B_{0}^{\top} x-H_{n} s=B_{0}^{\top}\left[x-B_{0}\left(B_{0}^{\top} B_{0}\right)^{-1} H_{n} s\right] \in \operatorname{supp}\left(B_{0}^{\top} X\right)$ since $\operatorname{supp}(X)$ is an open set, condition (10) entails that $f_{B_{0}^{\top} X}\left(B_{0}^{\top} x-H_{n} s\right) / f_{B_{0}^{\top} X}\left(B_{0}^{\top} x\right)-1=\mathcal{O}\left(\left\|H_{n}\right\|_{\infty}\right)=o\left(\tau_{n}\right)$ uniformly on $s \in \mathcal{U}_{q}$ and $x \in \operatorname{supp}(X)$. Moreover, using the first statement of Lemma 2, condition (12) entails that

$$
\frac{\mathbb{P}\left(Y>y_{n}(u \mid x) \mid B_{0}^{\top} X=B_{0}^{\top} x-H_{n} s\right)}{\mathbb{P}\left(Y>y_{n}(u \mid x) \mid B_{0}^{\top} X=B_{0}^{\top} x\right)}=1+\mathcal{O}\left(\tau_{n}\right),
$$

uniformly on $s \in \mathcal{U}_{q}$. As a first consequence,

$$
\mu_{n}(u \mid x) \sim n\left|H_{n}\right| f_{B_{0}^{\top} X}\left(B_{0}^{\top} x\right) \mathbb{P}\left(Y>y_{n}(u \mid x) \mid B_{0}^{\top} X=B_{0}^{\top} x\right) .
$$

First part of Lemma 2 entails that for all $\nu \in(0,1), \mu_{n}(\nu \mid x)$ is proportional to $n\left|H_{n}\right| \alpha_{n} \rightarrow \infty$ and that $\mu_{n}(\nu \mid x) / \mu_{n}(1 \mid x) \geq \nu / 2$ for $n$ large enough. Hence, condition (C.2) is satisfied. It also appears in turn that $v_{n, x}$ is asymptotically proportional to $\tau_{n}$ for all $x \in \operatorname{supp}(X)$. It remains to show (C.3). Let $\delta_{n}(u \mid x):=Q(0 \mid X=x)-y_{n}(u \mid x)$ if $Q(0 \mid X=x)<\infty$ and $\delta_{n}(u \mid x):=\left[y_{n}(u \mid x)\right]^{-1}$ if $Q(0 \mid X=x)=+\infty$. From the second statement of Lemma $2, \delta_{n}(u \mid x) \rightarrow 0$ uniformly on $u \in[\nu, 1]$. Hence, there exists $N_{x} \in \mathbb{N}$ such that for all $n \geq N_{x}, \delta_{n}(u \mid x) \in(0, \kappa]$ where $\kappa$ is such that (9) holds. Using (21) with $Z=B_{0}^{\top} X$ and under (9), one has for $n \geq N_{x}$

$$
\begin{aligned}
\left|\frac{\mathbb{P}\left(Y>y_{n}(u \mid x) \mid B_{0}^{\top} X=B_{0}^{\top} x\right)}{\mathbb{P}\left(Y>y_{n}(u \mid x) \mid X=x\right)}-1\right| & =\left|\frac{\mathbb{P}\left(Y>\mathcal{Y}_{\delta_{n}(u \mid x)}\left(B_{0}^{\top} x\right) \mid B_{0}^{\top} X=B_{0}^{\top} x\right)}{\mathbb{P}\left(Y>\mathcal{Y}_{\delta_{n}(u \mid x)}\left(B_{0}^{\top} x\right) \mid X=x\right)}-1\right| \\
& =\left|\frac{1+\mathbb{E}\left[\eta_{\delta_{n}(u \mid x)}(X) \mid B_{0}^{\top} X=B_{0}^{\top} x\right]}{1+\eta_{\delta_{n}(u \mid x)}(X=x)}-1\right| \\
& \leq \frac{2 \bar{\eta}\left(\delta_{n}^{-1}(u \mid x)\right)}{1-\bar{\eta}\left(\delta_{n}^{-1}(u \mid x)\right)} .
\end{aligned}
$$


Since $\bar{\eta}(\cdot)$ is a decreasing function, the second statement of Lemma 2 leads to $\bar{\eta}\left(\delta_{n}^{-1}(u \mid x)\right) \leq$ $\bar{\eta}\left(\xi \delta_{\alpha_{n}}^{-1}(X=x)\right)=\mathcal{O}\left(\tau_{n}\right)$ for all $x \in \mathcal{A}$. Hence, locally uniformly on $u \in(0, \infty)$,

$$
\frac{\mu_{n}(u \mid x)}{n\left|H_{n}\right| f_{B_{0}^{\top} X}\left(B_{0}^{\top} x\right) \mathbb{P}\left(Y>y_{n}(u \mid x) \mid X=x\right)}=1+\mathcal{O}\left(\tau_{n}\right) .
$$

Since $\tau_{n}^{-1} \operatorname{ERV}\left(\alpha_{n}, u \mid X=x\right) \rightarrow 0$ locally uniformly, one can use [16, Lemma 3] entailing that for $u \in[\nu, 1]$ and $u^{\prime} \in[\nu, 1]$ with $\left|u-u^{\prime}\right| \leq\left(\mu_{n}(1 \mid x)\right)^{-1 / 2}=o\left(\tau_{n}\right)$

$$
\begin{aligned}
& \alpha_{n}^{-1}\left[\mathbb{P}\left(Y>y_{n}(u \mid x) \mid X=x\right)-\mathbb{P}\left(Y>y_{n}\left(u^{\prime} \mid x\right) \mid X=x\right)\right] \\
& =\frac{1}{L_{\gamma(x)}^{\leftarrow}\left[L_{\gamma(x)}(1 / u)+o(1)\right]}-\frac{1}{L_{\gamma(x)}^{\leftarrow}\left[L_{\gamma(x)}\left(1 / u^{\prime}\right)+o(1)\right]}+o\left(\tau_{n}\right)
\end{aligned}
$$

where the terms in little-o do not depend on $u$ and $u^{\prime}$ and $L_{\gamma(x)}^{\leftarrow}(v):=(1+\gamma(x) v)^{1 / \gamma(x)}$. Since the derivatives of $1 / L_{\gamma(x)}^{\leftarrow}(\cdot)$ and $L_{\gamma(x)}(1 / \cdot)$ are bounded, it easy to check that (30) is a big-o of $\tau_{n}$ and thus, from (29), condition (C.3) is satisfied. Thus, the consistency result (28) is true. Using (29) and since $v_{n, x}$ is asymptotically proportional to $\tau_{n}$ for all $x \in \operatorname{supp}(X),(28)$ can be rewritten as

$$
\sup _{u \in[\nu, 1]}\left|\frac{\widehat{\Phi}_{n}(u \mid x)}{n\left|H_{n}\right| f_{B_{0}^{\top} X}\left(B_{0}^{\top} x\right) \mathbb{P}\left(Y>y_{n}(u \mid x) \mid X=x\right)}-1\right|=\mathcal{O}_{\mathbb{P}}\left(\tau_{n}\right) .
$$

Collecting (27) and (31) concludes the proof.

Proof of Theorem 2 - First, the consistency of $\widehat{\gamma}_{n}\left(B_{0}, x\right)$ is a direct consequence of [16, Theorem 1]. Indeed, under the assumptions of Theorem 2, Proposition 4 holds and thus assumptions of $[16$, Theorem 1] are satisfied leading to

$$
\widehat{\gamma}_{n}\left(B_{0}, x\right)-\gamma(x)=\mathcal{O}_{\mathbb{P}}\left(\tau_{n}\right) \text { and } \widehat{\gamma}_{n,-}\left(B_{0}, x\right)-\gamma_{-}(x)=\mathcal{O}_{\mathbb{P}}\left(\tau_{n}\right),
$$

almost everywhere for $x \in \operatorname{supp}(X)$. We are now interested in showing the consistency of the estimator $\widehat{a}_{n}\left(B_{0}, x\right)$. We have

$$
\frac{\widehat{a}_{n}\left(B_{0}, x\right)}{a\left(\alpha_{n}^{-1} \mid x\right)}=\frac{\widehat{Q}_{n}\left(\alpha_{n} \mid B_{0}, x\right)}{a\left(\alpha_{n}^{-1} \mid x\right)} \mathcal{T}_{\alpha_{n}}^{(1)}\left(\widehat{Q}_{n}\left(\cdot \mid B_{0}, x\right)\right) \int_{\nu}^{1} \varphi(u) L_{0}(1 / u) d u / \int_{\nu}^{1} \varphi(u) L_{\widehat{\gamma}_{n,-}\left(B_{0}, x\right)}(1 / u) d u
$$

Using the inequality $|1-\exp (x)| \leq|x|+x^{2}$ that holds for all $x<\ln (2)$, we have for all $u \in(\nu, 1)$,

$$
\begin{aligned}
& \left|L_{\widehat{\gamma}_{n,-}\left(B_{0}, x\right)}(1 / u)-L_{\gamma_{-}(x)}(1 / u)\right| \leq \int_{1}^{1 / \nu} v^{\gamma_{-}(x)-1}\left|\exp \left[\left(\widehat{\gamma}_{n,-}\left(B_{0}, x\right)-\gamma_{-}(x)\right) \ln (v)\right]-1\right| d v \\
\leq & \left|\widehat{\gamma}_{n,-}\left(B_{0}, x\right)-\gamma_{-}(x)\right| \tilde{L}_{\gamma_{-}(x)}(1 / \nu)\left(1+\ln ^{2}(\nu)\left|\widehat{\gamma}_{n,-}\left(B_{0}, x\right)-\gamma_{-}(x)\right|\right)=\mathcal{O}_{\mathbb{P}}\left(\tau_{n}\right),
\end{aligned}
$$

from (32). It is then straightforward to check that

$$
\int_{\nu}^{1} \varphi(u) L_{\widehat{\gamma}_{n,-}\left(B_{0}, x\right)}(1 / u) d u / \int_{\nu}^{1} \varphi(u) L_{\gamma_{n,-}(x)}(1 / u) d u=1+\mathcal{O}_{\mathbb{P}}\left(\tau_{n}\right) .
$$

As a consequence, since under the assumptions of Theorem 2, $\widehat{Q}_{n}\left(\alpha_{n} \mid B_{0}, x\right) / Q\left(\alpha_{n} \mid X=x\right)=$ $1+\mathcal{O}_{\mathbb{P}}\left(\tau_{n}\right)$, the estimator of $a(\cdot \mid x)$ is such that

$$
\frac{\widehat{a}_{n}\left(B_{0}, x\right)}{a\left(\alpha_{n}^{-1} \mid x\right)}=\frac{Q\left(\alpha_{n} \mid X=x\right)}{a\left(\alpha_{n}^{-1} \mid x\right)} \mathcal{T}_{\alpha_{n}}^{(1)}\left(\widehat{Q}_{n}\left(\cdot \mid B_{0}, x\right)\right) \frac{\int_{\nu}^{1} \varphi(u) L_{0}(1 / u) d u}{\int_{\nu}^{1} \varphi(u) L_{\gamma_{n,-}(x)}(1 / u) d u}\left(1+\mathcal{O}_{\mathbb{P}}\left(\tau_{n}\right)\right) .
$$

A direct consequence of the result established in [16, eq. (30)] leads to

$$
\widehat{a}_{n}\left(B_{0}, x\right)=a\left(\alpha_{n}^{-1} \mid x\right)\left(1+\mathcal{O}_{\mathbb{P}}\left(\tau_{n}\right)\right) .
$$


We are now in position to study the asymptotic behavior of $\check{Q}_{n}\left(\cdot \mid B_{0}, x\right)$. We start with

$$
\begin{aligned}
& \frac{\tau_{n}^{-1}}{a\left(\alpha_{n}^{-1} \mid X=x\right) \tilde{L}_{\gamma(x)}\left(\alpha_{n} / \beta_{n}\right)}\left|\check{Q}_{n}\left(\beta_{n} \mid B_{0}, x\right)-Q\left(\beta_{n} \mid X=x\right)\right| \\
\leq & \frac{1}{\tilde{L}_{\gamma(x)}\left(\alpha_{n} / \beta_{n}\right)} \frac{\left|\widehat{Q}_{n}\left(\alpha_{n} \mid B_{0}, x\right)-Q\left(\alpha_{n} \mid X=x\right)\right|}{\tau_{n} a\left(\alpha_{n}^{-1} \mid x\right)} \\
+ & \tau_{n}^{-1} \frac{\widehat{a}_{n}\left(B_{0}, x\right)}{a\left(\alpha_{n}^{-1} \mid x\right)} \frac{\left|L_{\widehat{\gamma}_{n}\left(B_{0}, x\right)}(1 / u)-L_{\gamma(x)}(1 / u)\right|}{\tilde{L}_{\gamma(x)}\left(\alpha_{n} / \beta_{n}\right)} \\
+ & \tau_{n}^{-1} \frac{L_{\gamma(x)}\left(\alpha_{n} / \beta_{n}\right)}{\tilde{L}_{\gamma(x)}\left(\alpha_{n} / \beta_{n}\right)}\left|\frac{\widehat{a}_{n}\left(B_{0}, x\right)}{a\left(\alpha_{n}^{-1} \mid x\right)}-1\right|+\frac{\tau_{n}^{-1}}{\tilde{L}_{\gamma(x)}\left(\alpha_{n} / \beta_{n}\right)} \operatorname{ERV}\left(\alpha_{n}, \beta_{n} / \alpha_{n} \mid X=x\right) \\
= & T_{1, n}(x)+T_{2, n}(x)+T_{3, n}(x)+T_{4, n}(x) .
\end{aligned}
$$

As a direct consequence of Proposition 4 (see $(26)), T_{1, n}(x)=\mathcal{O}_{\mathbb{P}}\left(1 / \tilde{L}_{\gamma(x)}\left(\alpha_{n} / \beta_{n}\right)\right)=\mathcal{O}_{\mathbb{P}}(1)$ since, as $t \rightarrow \infty$,

$$
\tilde{L}_{s}(t) \sim \begin{cases}t^{s} \ln (t) / s & \text { if } s>0 \\ \ln ^{2}(t) / 2 & \text { if } s=0 \\ 1 / s^{2} & \text { if } s<0\end{cases}
$$

Mimicking the proof of (33), since $\tau_{n} \ln ^{2}\left(\alpha_{n} / \beta_{n}\right) \rightarrow 0$ and using (34) lead to $T_{2, n}(x)=(1+$ $\left.\mathcal{O}_{\mathbb{P}}\left(\tau_{n}\right)\right) \mathcal{O}_{\mathbb{P}}\left[\tau_{n}^{-1}\left(\widehat{\gamma}_{n}\left(B_{0}, x\right)-\gamma(x)\right)\right]=\mathcal{O}_{\mathbb{P}}(1)$. Now, since for all $s \in \mathbb{R}, L_{s}(t) / \tilde{L}_{s}(t)=\mathcal{O}(1)$ as $t \rightarrow \infty$, equation (34) entails that $T_{3, n}(x)=\mathcal{O}_{\mathbb{P}}(1)$. Finally, since by assumption, $\tau_{n}^{-1} \operatorname{ERV}\left(\alpha_{n}, \alpha_{n} / \beta_{n} \mid X=\right.$ $x) / \tilde{L}_{\gamma(x)}\left(\alpha_{n} / \beta_{n}\right) \rightarrow 0$, it is clear that $T_{4, n}(x)=\mathcal{O}_{\mathbb{P}}(1)$ and the proof is complete.

Proof of Proposition 3 - Let $B_{0} \in \mathcal{B}$ and $B_{1} \in \mathcal{B}$ two $p \times q$ matrices of rank $q$ with $B_{0} \neq B_{1}$. Assume that $\mathcal{S}\left(B_{0}\right)$ and $\mathcal{S}\left(B_{1}\right)$ are minimum TDR subspaces. Let us first introduce the linear space $E:=\mathcal{S}\left(B_{0}\right) \cap \mathcal{S}\left(B_{1}\right)$. We assume that $E=\mathcal{S}(C)$ where $C$ is a $p \times r$ matrix with $r \in\{0, \ldots, q\}$. Note that if $r=q$ then $B_{0}=B_{1}$ and the result is proved. From now on, we assume that $r<q$. There thus exist two $p \times(q-r)$ matrices $D_{0}$ and $D_{1}$ such that $\mathcal{S}\left(B_{0}\right)=\mathcal{S}\left(D_{0}, C\right)$ and $\mathcal{S}\left(B_{1}\right)=\mathcal{S}\left(D_{1}, C\right)$. Since $\mathcal{S}\left(B_{0}\right)$ and $\mathcal{S}\left(B_{1}\right)$ are TDR subspaces, there exists a Borel set $\mathcal{A}$ of $\mathbb{R}^{p}$ with $\mathbb{P}(X \in \mathcal{A})=1$ and such that for all $x \in \mathcal{A}$,

$$
\lim _{\alpha \rightarrow 0} \frac{Q\left(\alpha \mid B_{0}^{\top} X=B_{0}^{\top} x\right)}{Q\left(\alpha \mid B_{1}^{\top} X=B_{1}^{\top} x\right)}=1 .
$$

Now, for a given $x^{*} \in \mathcal{A}$, let $E^{*}$ be the linear space given by $\left\{x \in \mathbb{R}^{p} \mid B_{0}^{\top} x=B_{0}^{\top} x^{*}\right\}$. It is clear that for all $x \in E^{*}, C^{\top} x=C^{\top} x^{*}$ and thus that

$$
\lim _{\alpha \rightarrow 0} \frac{Q\left(\alpha \mid B_{0}^{\top} X=D_{0}^{\top} x^{*}+C^{\top} x^{*}\right)}{Q\left(\alpha \mid B_{1}^{\top} X=D_{1}^{\top} x+C^{\top} x^{*}\right)}=1,
$$

for all $x \in E^{*}$. Since $\mathbb{P}\left(X \in\left\{D_{1}^{\top} x ; x \in E^{*}\right\}\right)=1$, it appears that, almost surely, the conditional quantile $Q\left(\alpha \mid B_{1}^{\top} X=D_{1}^{\top} x+C^{\top} x^{*}\right)$ is constant in $D_{1}^{\top} x$. As a consequence, for all $x \in \mathcal{A}$

$$
\lim _{\alpha \rightarrow 0} \frac{Q\left(\alpha \mid C^{\top} X=C^{\top} x\right)}{Q(\alpha \mid X=x)}=1,
$$

which is in contradiction with the fact that $\mathcal{S}\left(B_{0}\right)$ is a minimum TDR subspace (since $C$ is a matrix of $\operatorname{rank} r<q)$.

Proof of Theorem 3 - First remark that for all $x \in \operatorname{supp}(X)$,

$$
\alpha^{-1} \mathbb{P}\left[Y>Q\left(\alpha \mid B_{0}^{\top} X=B_{0}^{\top} x\right) \mid X=x\right]=\frac{\mathbb{P}\left[Y>\mathcal{Y}_{\delta_{\alpha, x}}\left(B_{0}^{\top} X=B_{0}^{\top} x\right) \mid X=x\right]}{\mathbb{P}\left[Y>\mathcal{Y}_{\delta_{\alpha, x}}\left(B_{0}^{\top} X=B_{0}^{\top} x\right) \mid B_{0}^{\top} X=B_{0}^{\top} x\right]},
$$


where, in order to not overload the notations, $\delta_{\alpha, x}$ stands for $\delta_{\alpha}\left(B_{0}^{\top} X=B_{0}^{\top} x\right)$. By assumption, there exists a Borel set $\mathcal{A} \in \mathcal{B}\left(\mathbb{R}^{p}\right)$ with $\mathbb{P}(X \in \mathcal{A})=1$ such that for all $x \in \mathcal{A}$ and $\kappa>0$, there exists $\alpha_{0} \in(0,1)$ such that for all $\alpha \in\left(0, \alpha_{0}\right), \delta_{\alpha, x}<\kappa$. Hence, since $\mathcal{S}\left(B_{0}\right)$ is a TDR subspace, for all $\varepsilon>0$, there exists $\alpha_{0} \in(0,1)$ such that for $\alpha \in\left(0, \alpha_{0}\right)$,

$$
\mathbb{P}\left[\left|\alpha^{-1} \mathbb{P}\left[Y>Q\left(\alpha \mid B_{0}^{\top} X\right) \mid X\right]-1\right|<\varepsilon\right]=1 .
$$

Mimicking the proof of the second statement of Theorem 1 and introducing for all $B \in \mathcal{B}$ and $j \in\{1, \ldots, J\}$ the quantity

$$
\Delta^{(j)}(\alpha, B):=\frac{\mathbb{P}\left[\left\{Y>Q\left(\alpha \mid B^{\top} X\right)\right\} \cap\left\{X \in \Pi_{B}^{(j)}\left(B^{\top} X\right)\right\} \mid B^{\top} X\right]}{\alpha \mathbb{P}\left[X \in \Pi_{B}^{(j)}\left(B^{\top} X\right) \mid B^{\top} X\right]}-1,
$$

one has that almost surely,

$$
\Delta^{(j)}\left(\alpha, B_{0}\right)=\mathbb{E}\left[\left(\frac{\mathbb{P}\left[Y>Q\left(\alpha \mid B_{0}^{\top} X\right) \mid X\right]}{\alpha}-1\right) \frac{\mathbb{I}_{\left\{X \in \Pi_{B_{0}}^{(j)}\left(B_{0}^{\top} X\right)\right\}}}{\mathbb{P}\left[X \in \Pi_{B_{0}}^{(j)}\left(B_{0}^{\top} X\right) \mid B_{0}^{\top} X\right]} \mid B_{0}^{\top} X\right] .
$$

Collecting (35) and (36) show that for all $\varepsilon \in(0,1)$, there exists $\alpha_{0} \in(0,1)$ such that for $\alpha \in\left(0, \alpha_{0}\right]$,

$$
T\left(\alpha, B_{0}\right)=\sum_{j=1}^{J}\left\{\mathbb{E}\left[\Delta^{(j)}\left(\alpha, B_{0}\right)\right]\right\}^{2} \leq J \varepsilon^{2}
$$

As a first conclusion, we have proved that $T\left(\alpha, B_{0}\right) \rightarrow 0$ as $\alpha \rightarrow 0$. Now, since under the assumptions of Theorem 3, a minimum TDR subspace is unique (see Proposition 3), and since $\left|\alpha^{-1} \mathbb{P}\left(Y>Q\left(\alpha \mid B^{\top} X\right) \mid X\right)-1\right|$ converges to a limit in $[0, \infty]$ uniformly on $B \in \mathcal{B}$ as $\alpha \rightarrow 0$, it is easy to check starting from (36) that for all $\varepsilon>0$ and $\eta>0$, there exists $\alpha_{\varepsilon, \eta} \in(0,1)$ such that for all $\alpha \in\left(0, \alpha_{\varepsilon, \eta}\right]$,

$$
\sup _{B \in \mathcal{B},\left\|B-B_{0}\right\| \geq \varepsilon} T(\alpha, B)>\eta .
$$

Assume that $\left\|\tilde{B}_{0}(\alpha)-B_{0}\right\|$ does not converges to 0 as $\alpha \rightarrow 0$. One can thus find $\varepsilon>0$ such that for all $\alpha_{0} \in(0,1)$, there exists $\alpha \in\left(0, \alpha_{0}\right]$ such that $\left\|\tilde{B}_{0}(\alpha)-B_{0}\right\|>\varepsilon$. Hence, for all $\eta>0$ and for all $\alpha_{0} \in\left(0, \alpha_{\varepsilon, \eta}\right]$, there exists $\alpha \in\left(0, \alpha_{0}\right]$ such that $T\left(\alpha, \tilde{B}_{0}(\alpha)\right)>\eta$. This point is obviously in contradiction with the fact that $T\left(\alpha, \tilde{B}_{0}(\alpha)\right) \leq T\left(\alpha, B_{0}\right) \rightarrow 0$, as $\alpha \rightarrow 0$.

\section{Appendix A - Additional results}

The aim of the next result is to rephrase condition (12) appearing in Theorem 2 in term of the conditional distribution of $Y$ given $X$.

Lemma 3. Assume that there exists a full rank matrix $B_{0}$ such that $\mathcal{S}\left(B_{0}\right)$ is a TDR subspace (i.e. for all $\delta>0, \mathbb{P}\left(Y>\mathcal{Y}_{\delta}\left(B_{0}^{\top} X\right) \mid X\right)=s_{\delta}\left(B_{0}^{\top} X\right)\left(1+\eta_{\delta}(X)\right)$ a.s. where $s_{\delta}\left(B_{0}^{\top} X=\cdot\right.$ ) and $\eta_{\delta}(X=\cdot)$ are measurable functions) and that condition (5) hold. For $(x, t) \in \operatorname{supp}(X) \times \mathbb{R}^{q}$ and $\zeta \in(0,1)$, let

$$
\tilde{\delta}_{\zeta}(t, x):= \begin{cases}Q\left(0 \mid X=B_{0}\left(B_{0}^{\top} B_{0}\right)^{-1} t\right)-Q(\zeta \mid X=x) & \text { if } Q(0 \mid X=x)<\infty \\ 1 / Q(\zeta \mid X=x) & \text { if } Q(0 \mid X=x)=\infty .\end{cases}
$$


If $\left(\alpha_{n}\right)$ and $\left(H_{n}\right)$ are two sequences converging to 0 with $n\left|H_{n}\right| \alpha_{n} \rightarrow \infty$, if there exists $\xi \in(0,1)$ such that

$$
\max \left\{\sup _{(t, \zeta) \in A_{n}}\left|\frac{s_{\tilde{\delta}_{\zeta}(t, x)}\left(B_{0}^{\top} X=t\right)}{s_{\tilde{\delta}_{\zeta}\left(B_{0}^{\top} x, x\right)}\left(B_{0}^{\top} X=B_{0}^{\top} x\right)}-1\right| ; \bar{\eta}\left(\xi \delta_{\alpha_{n}}^{-1}(X=x)\right)\right\}=\mathcal{O}\left(\tau_{n}\right),
$$

where $A_{n}=D\left(B_{0}^{\top} x, H_{n}\right) \times\left[\xi \nu \alpha_{n}, \xi^{-1} \alpha_{n}\right]$ and if for all $\varepsilon>0$, there exists $N \in \mathbb{N}$ such that for $n \geq N$,

$$
\begin{cases}\inf _{t \in D\left(B_{0}^{\top} x, H_{n}\right)} Q\left(0 \mid X=B_{0}\left(B_{0}^{\top} B_{0}\right)^{-1} t\right)=\infty & \text { if } Q(0 \mid X=x)=\infty \\ \sup _{t \in D\left(B_{0}^{\top} x, H_{n}\right)}\left|Q\left(0 \mid X=B_{0}\left(B_{0}^{\top} B_{0}\right)^{-1} t\right)-Q(0 \mid X=x)\right| / a\left(\alpha_{n}^{-1} \mid x\right)<\varepsilon & \text { if } Q(0 \mid X=x)<\infty\end{cases}
$$

then, there exists a Borel set $\mathcal{A} \in \mathcal{B}\left(\mathbb{R}^{p}\right)$ with $\mathbb{P}(X \in \mathcal{A})=1$ such that for all $x \in \mathcal{A}$,

$$
\sup _{(t, \zeta) \in A_{n}}\left|\frac{\mathbb{P}\left(Y>Q\left(\zeta \mid B_{0}^{\top} X=B_{0}^{\top} x\right) \mid B_{0}^{\top} X=t\right)}{\zeta}-1\right|=\mathcal{O}\left(\tau_{n}\right) .
$$

Proof - We start with the fact that for all $\delta>0$ and $t \in \operatorname{supp}\left(B_{0}^{\top} X\right)$,

$$
\mathbb{P}\left(Y>\mathcal{Y}_{\delta}(t) \mid B_{0}^{\top} X=t\right)=s_{\delta}\left(B_{0}^{\top} X=t\right)\left\{1+\mathbb{E}\left[\eta_{\delta}(X) \mid B_{0}^{\top} X=t\right]\right\} .
$$

For $\zeta \in(0,1)$, let

$$
\check{\delta}_{0, \zeta}(t, x):= \begin{cases}Q\left(0 \mid X=B_{0}\left(B_{0}^{\top} B_{0}\right)^{-1} t\right)-Q\left(\zeta \mid B_{0}^{\top} X=B_{0}^{\top} x\right) & \text { if } Q(0 \mid X=x)<\infty, \\ 1 / Q\left(\zeta \mid B_{0}^{\top} X=B_{0}^{\top} x\right) & \text { if } Q(0 \mid X=x)=\infty .\end{cases}
$$

Since $\mathcal{S}\left(B_{0}\right)$ is a TDR subspace one has that $Q\left(0 \mid B_{0}\left(B_{0}^{\top} B_{0}\right)^{-1} t\right)=Q\left(0 \mid B_{0}^{\top} X=B_{0}^{\top} x\right)$ almost surely and thus,

$$
\begin{aligned}
\frac{\mathbb{P}\left(Y>Q\left(\zeta \mid B_{0}^{\top} X=B_{0}^{\top} x\right) \mid B_{0}^{\top} X=t\right)}{\zeta} & =\frac{s_{\check{\delta}_{0, \zeta}(t, x)}\left(B_{0}^{\top} X=t\right)}{s_{\check{\delta}_{0, \zeta}\left(B_{0}^{\top} x, x\right)}\left(B_{0}^{\top} X=B_{0}^{\top} x\right)} \\
& \times \frac{1+\mathbb{E}\left[\eta_{\check{\delta}_{0, \zeta}(t, x)}(X) \mid B_{0}^{\top} X=t\right]}{1+\mathbb{E}\left[\eta_{\check{\delta}_{0, \zeta}\left(B_{0}^{\top} x, x\right)}(X) \mid B_{0}^{\top} X=B_{0}^{\top} x\right]}
\end{aligned}
$$

Let us focus on the first factor of (39). Under (5) and since $\mathcal{S}\left(B_{0}\right)$ is a TDR subspace, using [22, Lemma 1.2.12], it is easy to check that there exists a Borel set $\mathcal{A} \in \mathcal{B}\left(\mathbb{R}^{p}\right)$ with $\mathbb{P}(X \in \mathcal{A})=1$ such that for all $x \in \mathcal{A}$ and uniformly on $\zeta \in\left[\xi \nu \alpha_{n}, \xi^{-1} \alpha_{n}\right]$,

$$
\begin{aligned}
Q\left(\zeta \mid B_{0}^{\top} X=B_{0}^{\top} x\right) & =Q(\zeta \mid X=x)+a\left(\alpha_{n}^{-1} \mid x\right)\left(\frac{Q\left(\zeta \mid B_{0}^{\top} X=B_{0}^{\top} x\right)-Q(\zeta \mid X=x)}{a\left(\alpha_{n}^{-1} \mid x\right)}\right) \\
& =Q(\zeta \mid X=x)+o\left(a\left(\alpha_{n}^{-1} \mid x\right)\right) .
\end{aligned}
$$

Hence, mimicking the proof of the first statement of Lemma 2, we know that there exists $\tilde{\xi} \in(0,1)$ such that $Q\left(\zeta \mid B_{0}^{\top} X=B_{0}^{\top} x\right)=Q(\tilde{\zeta} \mid X=x)$ where $\tilde{\zeta} \in\left[\tilde{\xi} \zeta, \tilde{\xi}^{-1} \zeta\right]$. Hence $\check{\delta}_{0, \zeta}(t, x)=\tilde{\delta}_{\tilde{\zeta}}(t, x)$ and, by assumption, one can find $\xi \in(0,1)$ such that

$$
\sup _{(t, \zeta) \in A_{n}}\left|\frac{s_{\check{\delta}_{0, \zeta}(t, x)}\left(B_{0}^{\top} X=t\right)}{s_{\check{\delta}_{0, \zeta}\left(B_{0}^{\top} x, x\right)}\left(B_{0}^{\top} X=B_{0}^{\top} x\right)}-1\right|=\mathcal{O}\left(\tau_{n}\right)
$$

for all $x \in \mathcal{A}$.

Let us now consider the second factor of (39). We have shown before that $\check{\delta}_{0, \zeta}(t, x)=\tilde{\delta}_{\tilde{\zeta}}(t, x)$. 
Hence, if $Q(0 \mid X=x)<\infty$, under (38), one has for all $t \in D\left(B_{0}^{\top} x, H_{n}\right)$ that $\check{\delta}_{0, \zeta}(t, x)=Q(0 \mid X=$ $x)-\left[Q(\tilde{\zeta} \mid X=x)-o\left(a_{n}\left(\alpha_{n}^{-1} \mid x\right)\right)\right]$ and if $Q(0 \mid X=x)=\infty, \check{\delta}_{0, \zeta}(t, x)=1 / Q(\tilde{\zeta} \mid X=x)$. Using the second statement of Lemma 2 , one can find $\xi \in(0,1)$ such that $\check{\delta}_{0, \zeta}(t, x) \leq \xi^{-1} \delta_{\alpha_{n}}(X=x)$ uniformly on $t \in D\left(B_{0}^{\top} x, H_{n}\right)$. Applying condition (9) and since $\bar{\eta}\left(\xi \delta_{\alpha_{n}}^{-1}(X=x)\right)=\mathcal{O}\left(\tau_{n}\right)$, leads to

$$
\frac{1+\mathbb{E}\left[\eta_{\check{\delta}_{0, \beta}(t, x)}(X) \mid B_{0}^{\top} X=t\right]}{1+\mathbb{E}\left[\eta_{\check{\delta}_{0, \beta}\left(B_{0}^{\top} x, x\right)}(X) \mid B_{0}^{\top} X=B_{0}^{\top} x\right]}=\mathcal{O}\left(\tau_{n}\right) .
$$

Collecting (39), (40) and (41) concludes the proof.

The aim of the following lemma is to study the distributions introduced in Section 4.2.

Lemma 4. Using same notations as in Section 4.2, one has for all $x \in \mathbb{R}^{p}$ and $\delta>0$, (i) $S_{1}\left(\delta^{-1} \mid x\right)=s_{\delta, 1}\left(B_{0}^{\top} X=B_{0}^{\top} x\right)\left[1+\eta_{\delta, 1}(X=x)\right]$ with $s_{\delta, 1}\left(B_{0}^{\top} X=B_{0}^{\top} x\right)=\delta^{1 / g_{0}\left(B_{0}^{\top} x\right)}$ and for $\delta \in[0,1],\left|\eta_{\delta, 1}(X=x)\right| \leq \bar{\eta}_{1}\left(\delta^{-1}\right)$ where $\bar{\eta}_{1}(\cdot)$ is a decreasing function converging to 0 at infinity and defined for $y>1$ by $\bar{\eta}_{1}(y)=1-y^{-1 / 3}\left[\ln \left(1 /\left(1-\delta^{-1 / 3}\right)\right]^{-1}\left[1+\exp \left(10-y^{1 / 3}\right]^{-3}\right.\right.$.

(ii) $S_{2}\left(g_{2}\left(B_{0}^{\top} x\right)-\delta \mid x\right)=S_{1}\left(\delta^{-1} \mid x\right)$.

(iii) $S_{3}\left(\delta^{-1} \mid x\right)=\exp \left[-\delta^{-1 / g_{0}\left(B_{0}^{\top} x\right)} L_{3}\left(\delta^{-1} \mid x\right)\right]$ where $L_{3}(\cdot \mid x)$ converges to 1 at infinity. Furthermore, $S_{3}\left(\delta^{-1} \mid x\right)=s_{\delta, 3}\left(B_{0}^{\top} X=B_{0}^{\top} x\right)\left[1+\eta_{\delta, 3}(X=x)\right]$ with $s_{\delta, 3}\left(B_{0}^{\top} X=B_{0}^{\top} x\right)=\exp \left[-\delta^{-1 / g_{0}\left(B_{0}^{\top} x\right)}\right]$ and for $\delta \in[0,1],\left|\eta_{\delta, 3}(X=x)\right| \leq \bar{\eta}_{3}\left(\delta^{-1}\right)$ where $\bar{\eta}_{3}(\cdot)$ is a decreasing function converging to 0 at infinity and defined for $y>1$ by $\bar{\eta}_{3}(y)=1-\exp \left\{y^{3}\left[1-\left(1+\exp \left(10-\exp \left(y^{1 / 3}\right)\right)^{3}\right]\right\}\right.$.

Proof - (i) First, remark that for $\alpha \in[0,1]$ and $x \in \mathbb{R}^{p}, Q_{1}(\alpha \mid X=x)=\alpha^{-g_{0}\left(B_{0}^{\top} x\right)} \tilde{L}_{1}(\alpha \mid x)$, where $\tilde{L}_{1}(\alpha \mid x)=\alpha^{g_{0}\left(B_{0}^{\top} x\right)}\left[\ln (1 /(1-\alpha)]^{-g_{0}\left(B_{0}^{\top} x\right)}\left[1+g_{1}\left(B_{0}^{\top} x\right) \exp \left(-\alpha^{-1}\right)\right]^{-1}\right.$. Since the distribution function of $Y$ given $X=x$ is continuous, $Q_{1}\left(S_{1}\left(\delta^{-1} \mid x\right) \mid X=x\right)=\delta^{-1}$ for all $\delta>0$ and thus,

$$
S_{1}\left(\delta^{-1} \mid x\right)=\delta^{1 / g_{0}\left(B_{0}^{\top} x\right)}\left[\tilde{L}_{1}\left(S_{1}\left(\delta^{-1} \mid x\right) \mid x\right)\right]^{1 / g_{0}\left(B_{0}^{\top} x\right)} .
$$

It is quite easy to check that on $[0,1], \tilde{L}_{1}(\cdot \mid x)$ is a decreasing function with $\tilde{L}_{1}(\alpha \mid x) \rightarrow 1$ as $\alpha \rightarrow 0$. Hence, (42) entails that for $\delta \in[0,1], S_{1}\left(\delta^{-1} \mid x\right) \leq \delta^{1 / g_{0}\left(B_{0}^{\top} x\right)} \leq \delta^{1 / 3}$ since $g_{0}\left(B_{0}^{\top} x\right) \in[1 / 3,3]$. As a consequence, for $\delta \in[0,1]$,

$$
\left|\eta_{\delta, 1}(X=x)\right|=1-\left[\tilde{L}_{1}\left(S_{1}\left(\delta^{-1} \mid x\right) \mid x\right)\right]^{1 / g_{0}\left(B_{0}^{\top} x\right)} \leq 1-\left[\tilde{L}_{1}\left(\delta^{1 / 3} \mid x\right)\right]^{3} \leq \bar{\eta}_{1}\left(\delta^{-1}\right),
$$

since $g_{0}\left(B_{0}^{\top} x\right) \in[1 / 3,3]$ and $g_{1}\left(B_{0}^{\top} x\right) \in[1, \exp (10)]$.

(ii) This is a direct consequence of the equality $Q_{2}(\alpha \mid X=x)=g_{2}\left(B_{0}^{\top} x\right)-\left[Q_{1}(\alpha \mid X=x)\right]^{-1}$.

(iii) Since the distribution function of $Y$ given $X=x$ is continuous, $Q_{3}\left(S_{3}\left(\delta^{-1} \mid x\right) \mid X=x\right)=\delta^{-1}$ for all $\delta>0$ and thus, $S_{3}\left(\delta^{-1} \mid x\right)=\exp \left[-\delta^{-1 / g_{0}\left(B_{0}^{\top} x\right)} \tilde{L}_{3}\left(S_{3}\left(\delta^{-1} \mid x\right) \mid x\right)\right]$ with $\tilde{L}_{3}(\alpha \mid x)=[1+$ $\left.g_{1}\left(B_{1}^{\top} x\right) \exp \left(-\alpha^{-1}\right)\right]^{1 / g_{0}\left(B_{0}^{\top} x\right)}$. Obviously, $L_{3}(\cdot \mid x):=\tilde{L}_{3}\left(S_{3}(\cdot \mid x) \mid x\right)$ converges to 1 at infinity. Furthermore, $S_{3}\left(\delta^{-1} \mid x\right)=\exp \left[-\delta^{-1 / g_{0}\left(B_{0}^{\top} x\right)}\right]\left[1+\eta_{\delta, 3}(X=x)\right]$ with

$$
\eta_{\delta, 3}(X=x)=\exp \left\{\delta^{-1 / g_{0}\left(B_{0}^{\top} x\right)}\left[1-\tilde{L}_{3}\left(S_{3}\left(\delta^{-1} \mid x\right) \mid x\right)\right]\right\}-1 .
$$

It is easy to check that on $[0,1], \tilde{L}_{3}(\cdot \mid x)$ is an increasing function larger than 1 . Thus, for all $\delta \in[0,1), S_{3}\left(\delta^{-1} \mid x\right) \leq \exp \left(-\delta^{-1 / g_{0}\left(B_{0}^{\top} x\right)}\right) \leq \exp \left(-\delta^{-1 / 3}\right)$ and hence,

$$
\begin{aligned}
\left|\eta_{\delta, 3}(X=x)\right| & =1-\exp \left\{\delta^{-1 / g_{0}\left(B_{0}^{\top} x\right)}\left[1-\tilde{L}_{3}\left(S_{3}\left(\delta^{-1} \mid x\right) \mid x\right)\right]\right\} \\
& \leq 1-\exp \left\{\delta^{-1 / g_{0}\left(B_{0}^{\top} x\right)}\left[1-\tilde{L}_{3}\left(\exp \left(-\delta^{-1 / 3}\right) \mid x\right)\right]\right\} \leq \bar{\eta}_{3}\left(\delta^{-1}\right)
\end{aligned}
$$

using the facts that $g_{0}\left(B_{0}^{\top} x\right) \in[1 / 3,3]$ and $g_{1}\left(B_{1}^{\top} x\right) \in[1, \exp (10)]$. 


\section{Appendix B - Figures and tables}
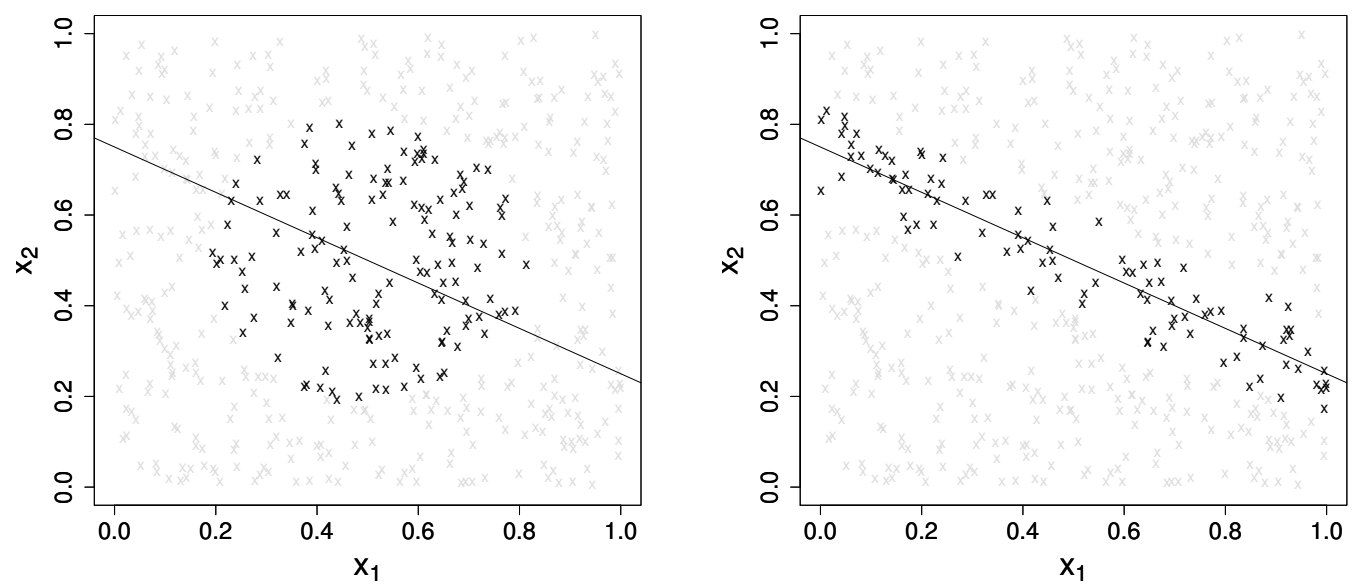

Figure 1: Representation of $n=500$ realizations of the random vector $X=\left(X_{1}, X_{2}\right)^{\top}$. The straight line is the set $\left\{x=\left(x_{1}, x_{2}\right)^{\top}, B_{0}^{\top} x=B_{0}^{\top} x_{0}\right\}$. The black points are the observations used to compute the classical estimator $\check{Q}_{n}\left(\cdot \mid x_{0}\right)$ (left) and $\check{Q}_{n}\left(\cdot \mid B_{0}, x_{0}\right)$ (right).

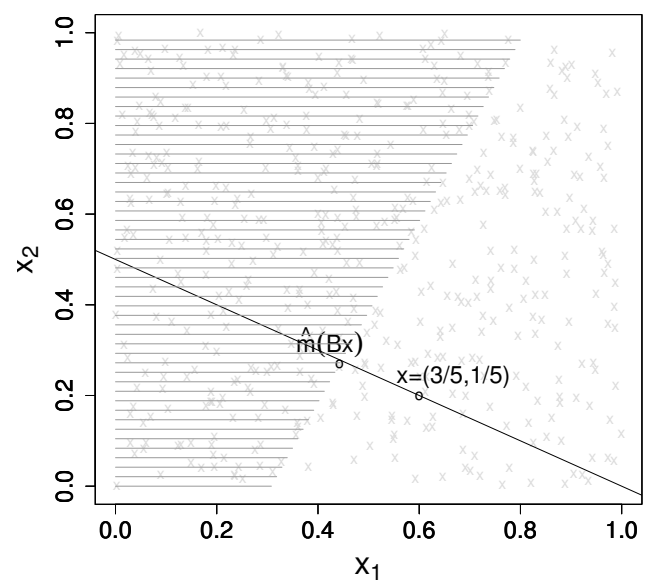

Figure 2: Representation of $n=500$ realizations of the random vector $X=\left(X_{1}, X_{2}\right)^{\top}$. The straight line is the set $\left\{s \in \mathbb{R}^{2}, B^{\top} s=B^{\top} x=1\right\}$ with $B=(1,2)^{\top} / \sqrt{5}$. The set $\Pi_{1}\left(B^{\top} X=B^{\top} x\right)$ is the hatched area, the rest is the set $\Pi_{2}\left(B^{\top} X=B^{\top} x\right)$. 


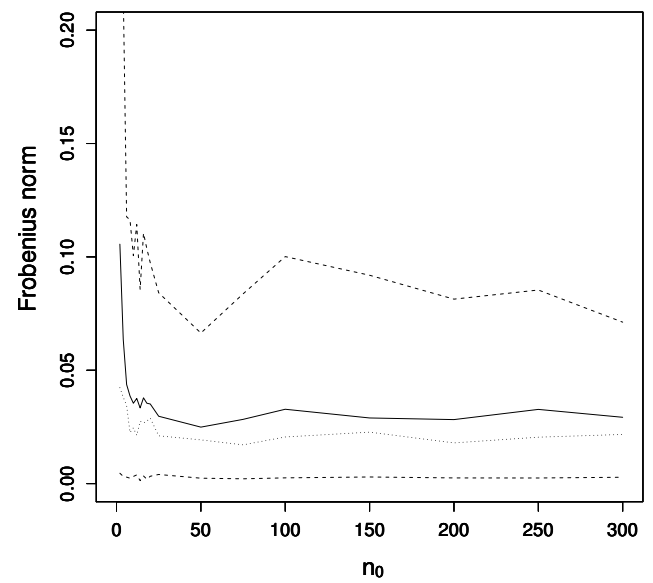

Figure 3: Under model 1 with $\sigma=1 / 5$, the empirical quantiles and the empirical mean of the $N=100$ obtained values of $\left\|\widehat{B}_{0, n}-B_{0}\right\|_{F}$ are represented as a function of $n_{0}$. The two dashed lines are the empirical quantiles of order 0.05 and 0.95 , the dotted line is the median and the full line is the empirical mean.
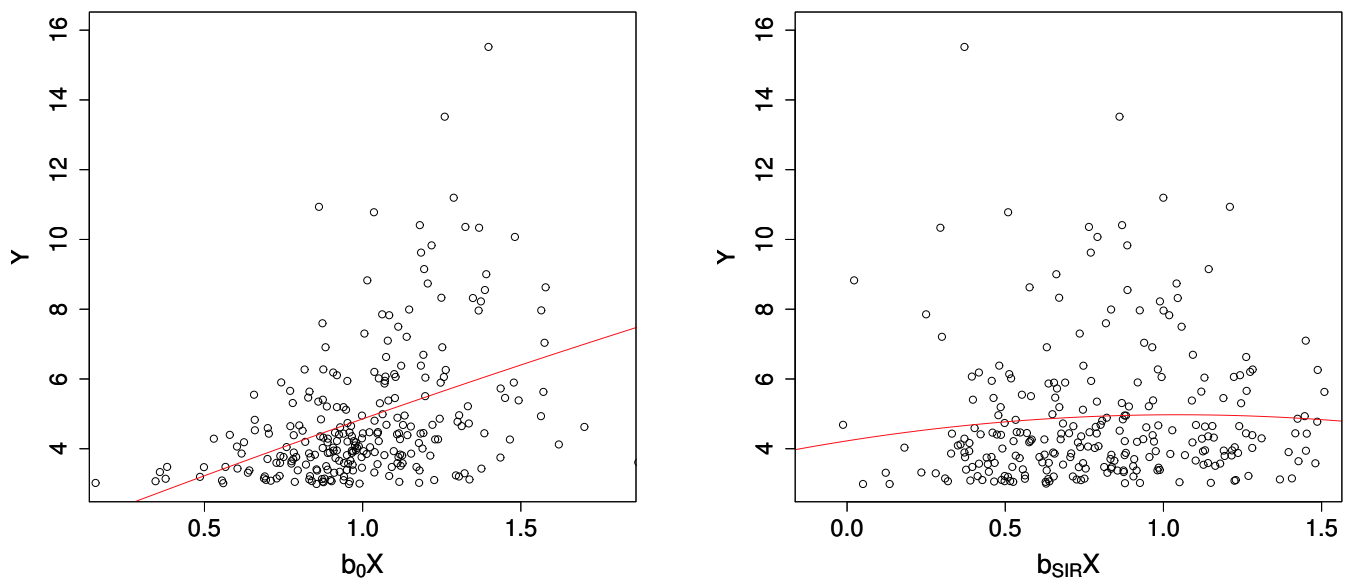

Figure 4: Scatter plots of the $\left(100 \times \alpha_{n}\right) \%$ largest observations of $Y$ (in a logarithmic scale) versus the corresponding projection on $\widehat{B}_{0, n}$ (left panel) and $\widehat{B}_{0, n}^{\mathrm{SIR}}$ (right panel). The full line is the polynomial fitting of order 2 . 


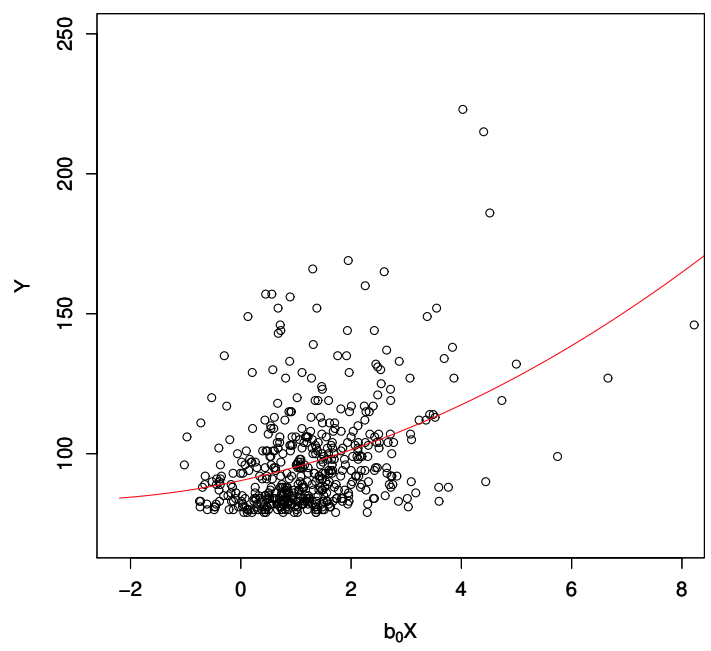

Figure 5: Scatter plot of the $\left(100 \times \hat{\alpha}_{n}\right) \%$ largest observations of $Y$ versus the corresponding projection on $\widehat{B}_{0, n}$. The full line is the polynomial fitting of order 2 .

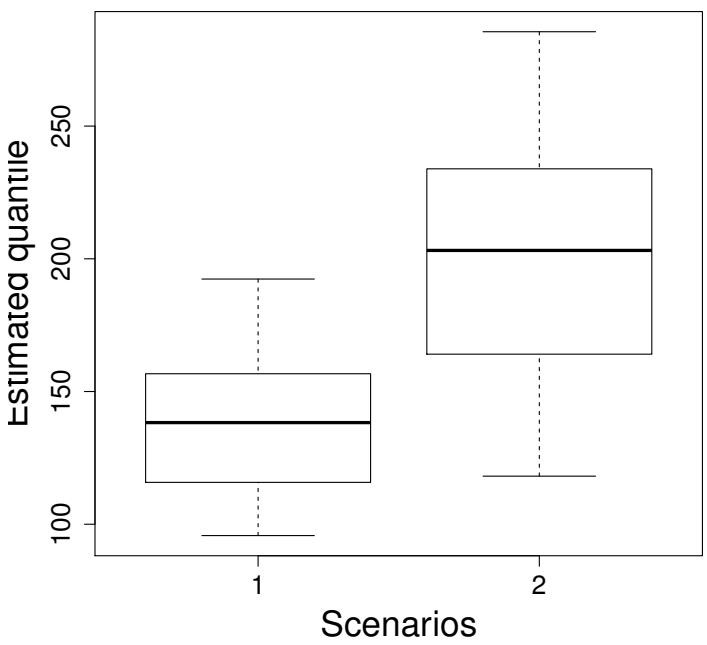

Figure 6: Box-plot of the jackknife sample distribution of the estimator $\check{Q}\left(1 / n \mid \widehat{B}_{0, n}, x\right)$ for the two scenarios. 


\begin{tabular}{|cc||ccccc|}
\hline & & $\left\|\widehat{B}_{0, n}-B_{0}\right\|_{F}$ & $\left\|\widehat{B}_{0, n}^{\mathrm{SIR}}-B_{0}\right\|_{F}$ & $\mathrm{E}_{Q}\left(\widehat{B}_{0, n}\right)$ & $\mathrm{E}_{Q}\left(B_{0}\right)$ & $\mathrm{E}_{Q}\left(I_{p}\right)$ \\
\hline \hline \multirow{2}{*}{ Model 1 } & $(1)$ & $0.031(0.100)$ & $1.759(0.048)$ & $0.966(1.787)$ & $0.571(0.411)$ & $6.966(2.801)$ \\
& $(2)$ & $0.029(0.025)$ & $1.751(0.058)$ & $0.841(0.491)$ & $0.672(0.540)$ & $4.353(0.900)$ \\
& $(3)$ & $0.061(0.053)$ & $1.734(0.082)$ & $1.977(1.304)$ & $1.631(1.333)$ & $5.253(0.377)$ \\
\hline \multirow{2}{*}{ Model 2 } & $(1)$ & $0.082(0.318)$ & $1.511(0.130)$ & $0.232(0.151)$ & $0.199(0.004)$ & $0.252(0.020)$ \\
& $(2)$ & $0.006(0.014)$ & $1.375(0.148)$ & $0.070(0.016)$ & $0.063(0.004)$ & $0.847(0.058)$ \\
& $(3)$ & $0.005(0.004)$ & $1.167(0.208)$ & $0.059(0.005)$ & $0.057(0.005)$ & $0.461(0.043)$ \\
\hline \multirow{2}{*}{ Model 3 3) } & $0.026(0.089)$ & $1.351(0.050)$ & $0.123(0.303)$ & $0.058(0.043)$ & $1.638(0.396)$ \\
& $(2)$ & $0.028(0.030)$ & $1.376(0.063)$ & $0.072(0.039)$ & $0.051(0.031)$ & $0.647(0.170)$ \\
& $(3)$ & $0.057(0.050)$ & $1.395(0.101)$ & $0.109(0.049)$ & $0.084(0.053)$ & $0.428(0.070)$ \\
\hline
\end{tabular}

Table 1: Estimation of the TDR direction and large conditional quantile of order $\beta_{n}=2 / n$ under models 1 to 3 with (1): $\sigma=1 / 3,(2): \sigma=1 / 5$ and (3): $\sigma=1 / 8$. The given values are the empirical means over the $N=100$ replications (the standard deviation is between brackets).

\begin{tabular}{|r||cc|}
\hline & $\left\|\widehat{B}_{0, n}-B_{0}\right\|_{F}$ & $\mathrm{E}_{Q}\left(\widehat{B}_{0, n}\right)$ \\
\hline \hline$(1)$ & $0.061(0.053)$ & $1.977(1.304)$ \\
$(2)$ & $0.068(0.059)$ & $4.175(2.250)$ \\
$(3)$ & $0.075(0.064)$ & $3.910(0.705)$ \\
$(4)$ & $1.769(0.176)$ & $19.75(1.976)$ \\
\hline \hline
\end{tabular}

Table 2: Estimation of the TDR direction and large conditional quantile of order $\beta_{n}=2 / n$ under model 1 with $\sigma=1 / 8$ and (1): $\alpha_{n}=n^{-1 / 3}, H_{n}=n^{-2 / 9},(2): \alpha_{n}=H_{n}=n^{-1 / 3},(3): \alpha_{n}=n^{-1 / 3}$, $H_{n}=n^{-1 / 10}$ and (4): $\alpha_{n}=H_{n}=n^{-1 / 10}$. The given values are the empirical means over the $N=100$ replications (the standard deviation is between brackets). 